\title{
All orders Boltzmann collision term from the multiple scattering expansion of the self-energy
}

\author{
François Fillion-Gourdeau* Jean-Sébastien Gagnon † and Sangyong Jeont \\ Department of Physics, McGill University, \\ 3600 University Street, Montreal, Canada H3A 278
}

(Dated: October 4, 2018)

\begin{abstract}
Starting from the Kadanoff-Baym relativistic transport equation and the multiple scattering expansion of the self-energy, we obtain the Boltzmann collision terms for any number of participating particles to all orders in perturbation theory within a quasi-particle approximation. This work completes a program initiated by Carrington and Mrówczyński and developed further by present authors and Weinstock in recent literature.
\end{abstract}

\section{INTRODUCTION}

The study of non-equilibrium phenomena is one of the most interesting problems in physics. In particular, the study of the formation and evolution of the quark gluon plasma (QGP) in heavy ion collisions is both theoretically and experimentally challenging. The current dynamical picture of the evolution of the "fireball" involves many stages. On one hand, it is clear from a theoretical point of view that the saturated gluon state (the so-called Color Glass Condensate) describes the initial states of the relativistic nuclei [1, 2, 3, 4, 5, 6, 4]. On the other hand, it is an experimental fact that the final states only consist of fully formed hadrons. In between these two extremes, the hot and dense matter evolves through many stages; after the initial collision, the quarks and gluons must scatter enough times to thermalize and form a QGP. This lump of QGP then must expand hydrodynamically, eventually reaching low enough temperatures to undergo a phase transition to hadronic matter. These hadrons interact further until the final kinetic freezout temperature is reached. None of these stages are static nor are they in global equilibrium.

In principle, quantum field theory is capable of explaining all of the above processes: Schwinger and Keldysh taught us how to formulate the problem a long time ago [8, 9]. Actually solving a non-equilibrium problem is, of course, a difficult task. For non-equilibrium

*Electronic address: ffillion@hep.physics.mcgill.ca

${ }^{\dagger}$ Electronic address: gagnonjs@physics.mcgill.ca

${ }_{\ddagger}^{\ddagger}$ Electronic address: jeon@physics.mcgill.ca 
problems, the most frequently used theoretical tools are linear response theory, hydrodynamics and kinetic theory. Linear response theory and hydrodynamics assume that the system under consideration is in a near-equilibrium state. In fact these two approaches are related in the sense that the transport coefficients needed in hydrodynamics can be calculated using linear response theory [15]. For these two approaches, their formalism is general and does not rely on perturbation theory. Although perturbative calculations are almost always the only practical way to calculate the transport coefficients, in principle the validity of these approaches depends only on the assumption that the system is near equilibrium.

Kinetic theory, in this sense, is different. A kinetic theory is a theory of colliding particles and as such, it must rely on the smallness of coupling constants [10, 11, 12, 13, 14]. First of all, to have well-defined particle degrees of freedom, collision rates must be small enough so that the final state particles of an individual collision have enough time to become on-shell (decohere) before being involved in the next collision. Second, the decay rate of the particles must be small enough so that the particles become on-shell first and then decay. The strong point of kinetic theory is that as long as these conditions are satisfied, one can numerically study arbitrarily out-of-equilibrium systems. The success of the so-called 'cascade models' or 'transport models' for modelling relativistic heavy ions collisions attests the strength of such an approach.

Current transport models rarely go beyond including $2 \rightarrow 2$ and $2 \rightarrow 3$ processes and resonance decays. In a recent paper, Carrington and Mrówczyński [16] addressed the multiparticle interactions in kinetic theories starting from the Schwinger-Keldysh formulation of quantum field theory (see also Ref.[17]). For a QGP and also for the early universe, we expect the systems to be dense enough for multi-particle interactions to become important. In Ref. 18] for example, it is argued that the thermalization of the fireball in their scenario would not be fast enough without $g g \rightarrow g g g$ scatterings. Other studies also stress the importance of multi-particle interactions (e.g. [14, 19, 20, 21]). In the context of transport coefficients, it has been shown that for bulk viscosity in hot scalar theories, number changing processes are essential and must be included [10, 11].

The method developped in Ref.[16] is therefore important because it provides a systematic way to obtain relativistic transport equations that include multi-particle interactions, starting from first principles. Using a $g \phi^{3}+\lambda \phi^{4}$ scalar field theory, these authors demonstrated that up to 4-loop self-energies, the collision term in the Kadanoff-Baym equation can be re-expressed as the usual gain and loss rates in a Boltzmann equation within a certain quasi-particle ansatz.

As the number of loops increases, the number of relevant diagrams increases very quickly. The authors of Ref.[16] managed this problem by developing a symbolic manipulation program to reorganize the diagrams in terms of vacuum scattering processes. The method could 
in principle be applied to any loops and any theories, but at higher orders, the task quickly becomes cumbersome.

The goal of this paper is to generalize the results of [16] using a different method. Instead of using a perturbative expansion, we use the multiple scattering expansion of the self-energy described in Ref. 22] to show that the pattern exhibited in Ref. 16] is in fact general.

A summary of our earlier work on this subject has been published in the proceedings of Quark Matter 2005 [23]. A similar work was also made public [24] shortly after, whose main results included only tree-level scattering matrix elements. The present contribution completes these works so that the analysis can be extended to all orders of perturbation theory involving any number of participating particles.

The rest of the paper is organized as follows. To set the notations, and also set the stage for the rest of the paper, we present in Section [1 a brief derivation of the KadanoffBaym equation for scalar field theories, emphasizing the approximations used. In Section III. we summarize the derivation of the multiple scattering expansion of the self-energy and introduce our quasi-particle ansatz. We present the derivation of the Boltzmann collision term in Section IV and finally conclude in Section $\mathrm{V}$

\section{KADANOFF-BAYM EQUATION}

The goal of this section is to briefly discuss the Kadanoff-Baym transport equation for a scalar field theory. Since there are many excellent papers and books on the subject (e.g. [16, 25, 26, 27, 28]), we do not attempt another derivation here. Instead we focus on the approximations and assumptions used in the derivation and the interpretations of the equation. The notations and discussions in this section largely follow those in Ref. [16] (with a different $i$ convention for propagators, however). The starting point is the following Lagrangian density for a real scalar field:

$$
\mathcal{L}(x)=\frac{1}{2} \partial^{\mu} \phi \partial_{\mu} \phi-\frac{1}{2} m^{2} \phi^{2}-\frac{g}{3 !} \phi^{3}-\frac{\lambda}{4 !} \phi^{4}
$$

To discuss the Kadanoff-Baym equation, it is convenient to define the following set of twopoint Green's functions:

$$
\begin{aligned}
& D_{O}^{c}(x, y) \equiv \theta\left(x_{0}-y_{0}\right)\left\langle\hat{O}_{H}(x) \hat{O}_{H}(y)\right\rangle+\theta\left(y_{0}-x_{0}\right)\left\langle\hat{O}_{H}(y) \hat{O}_{H}(x)\right\rangle \\
& D_{O}^{a}(x, y) \equiv \theta\left(y_{0}-x_{0}\right)\left\langle\hat{O}_{H}(x) \hat{O}_{H}(y)\right\rangle+\theta\left(x_{0}-y_{0}\right)\left\langle\hat{O}_{H}(y) \hat{O}_{H}(x)\right\rangle \\
& D_{O}^{>}(x, y) \equiv\left\langle\hat{O}_{H}(x) \hat{O}_{H}(y)\right\rangle \\
& D_{O}^{<}(x, y) \equiv\left\langle\hat{O}_{H}(y) \hat{O}_{H}(x)\right\rangle=D_{O}^{>}(y, x)
\end{aligned}
$$


Here $\hat{O}_{H}(x)$ is a Heisenberg picture operator and the average is defined with respect to the initial density operator $\langle\cdots\rangle \equiv \operatorname{Tr}\left(\hat{\rho}_{0} \cdots\right)$. The superscript $c(a)$ stands for chronological (anti-chronological) ordering. In the language of the Schwinger-Keldysh closed time path formalism, we have $D_{O}^{c}=D_{O}^{11}, D_{O}^{a}=D_{O}^{22}, D_{O}^{>}=D_{O}^{21}$ and $D_{O}^{<}=D_{O}^{12}$, where the superscripts 1 (2) refer to the time branch where the time goes from $-\infty$ to $+\infty(+\infty$ to $-\infty)$. It is also useful to define the retarded and advanced two-point Green's functions:

$$
\begin{aligned}
i D_{O}^{\mathrm{ret}}(x, y) & \equiv \theta\left(x^{0}-y^{0}\right)\left\langle\left[\hat{O}_{H}(x), \hat{O}_{H}(y)\right]\right\rangle \\
i D_{O}^{\mathrm{adv}}(x, y) & \equiv \theta\left(y^{0}-x^{0}\right)\left\langle\left[\hat{O}_{H}(y), \hat{O}_{H}(x)\right]\right\rangle=i D_{O}^{\mathrm{ret}}(y, x)
\end{aligned}
$$

In this paper, we will use the symbols $G^{<,>}, \ldots$ for propagators and $\Pi^{<,>}, \ldots$ for self-energies.

The derivation of the Kadanoff-Baym equation starts with the following Schwinger-Dyson equation for the contour propagator

$$
G(x, y)=G_{0}(x, y)+i \int_{C} d^{4} z \int_{C} d^{4} z^{\prime} G_{0}(x, y) \Pi\left(z, z^{\prime}\right) G\left(z^{\prime}, y\right)
$$

where the subscript 0 signifies free field quantities and the time contour runs from $-\infty$ to $\infty$ along the real time axis and then back to $-\infty$ slightly below the real time axis. Since we want to derive a transport equation, we need to turn this propagator equation into an equation for the distribution function $f(X, \mathbf{p})$. To do so, one first needs to turn Eq.(8) into an equation for the Wigner transformed $G^{<,>}$. Since the Wigner transform $G^{<,>}(X, p)$ is the quantum analog of the classical distribution function (when it is positive) [27, 28], we can make the following ansatz for our distribution function:

$$
\theta\left(p^{0}\right) G^{<}(X, p)=\theta\left(p^{0}\right) \rho(X, p) f(X, p)
$$

where $\rho(X, p)$ is the Wigner transformed spectral density

$$
\rho(X, p)=\int d^{4} u e^{i p u}\langle[\hat{\phi}(X+u / 2), \hat{\phi}(X-u / 2)]\rangle
$$

To make further progress, it is necessary to make approximations. More precisely, we require weak inhomogeneity of the system with respect to the inverse characteristic momentum (allowing for the gradient expansion) and the Compton wavelength (justifying the quasi-particle approximation) in addition to the weak couplings. These approximations are necessary for transport theory, and we will come back to them in more details in Section IIID Using these approximations and following the steps in [16], we get the Kadanoff-Baym equation for a scalar theory:

$$
E_{p}\left(\frac{\partial}{\partial t}+\mathbf{v} \cdot \nabla\right) f(X, \mathbf{p})+\nabla V(X, \mathbf{p}) \nabla_{p} f(X, \mathbf{p})=\theta\left(p_{0}\right) C(X, p)
$$


along with the mass-shell condition:

$$
p^{2}-m^{2}+V(X, p)=0
$$

where $V(X, p)=\Pi_{\delta}(X)+\operatorname{Re} \Pi^{\text {ret }}(X, p)$ is the Vlasov term (corresponding to an effective mass). Here $\Pi_{\delta}$ is the tadpole self-energy, $E_{p}$ is the solution of the mass-shell condition (12) and $\mathbf{v} \equiv \frac{\partial E_{p}}{\partial \mathbf{p}}$ is the velocity of propagation. The collision term is given by:

$$
C(X, p)=\frac{1}{2}\left[\Pi^{<}(X, p)(f(X, \mathbf{p})+1)-\Pi^{>}(X, p) f(X, \mathbf{p})\right]
$$

where $\Pi^{<,>}(X, p)$ are the Wightman self-energies. These Wightman functions are "completely cut" self-energies and can be interpreted as gain and loss rates. Written in this form, the transport equation is similar to the Boltzmann equation of classical kinetic theory. The collision term includes a gain term with an appropriate Bose-Einstein enhancement factor and a loss term [27].

The derivation of the collision term Eq.(13) in terms of the scattering processes is the main goal of this paper. The microscopic gain/loss rates themselves must be computed using the vaccum quantum field theory with the in-medium modified mass. The procedure followed in Ref. [16] is to directly analyze multi-loop (up to four) self-energy diagrams using a symbolic manipulation program and express them in terms of physical processes. In this paper, we use another method based on the multiple scattering expansion of the self-energy.

\section{SCATTERING EXPANSION OF THE SELF-ENERGY}

\section{A. Presentation of the Expansion}

The purpose of this section is to present the central ideas involved in the multiple scattering expansion of the self-energy. We will use it in Section IV to express the self-energies $\Pi^{<,>}(X, p)$ in terms of scattering amplitudes so that $C(X, p)$ in Eq.(11) can be interpreted as a kinetic theory collision term. For general equilibrium theories, the scattering expansion was accomplished in Ref. 22]. Here we repeat the central points and explain how it should be modified to deal with subtleties associated with medium complications.

In this section, we use equilibrium quantum field theory to derive the expansion. However, as long as we are careful and do not use the KMS condition, the results in this section can be readily generalized to the non-equilibrium case by a simple ansatz $n\left(E_{p}\right) \rightarrow f(X, \mathbf{p})$, where $f(X, \mathbf{p})$ is the non-equilibrium phase space density. This is a very simple extension of the equilibrium theory to the non-equilibrium case; but as long as the Kadanoff-Baym equation is valid, it captures a large amount of physics. 
The starting point of our derivation is the four propagators ${ }^{1}$ corresponding to the Green functions in the Schwinger-Keldysh contour. Denoting the regular $(-\infty, \infty)$ time branch as "1" and the reverse $(\infty,-\infty)$ branch as " 2 " , the free propagators for equilibrium situations are given by $[22,29]$ :

$$
\begin{aligned}
& G_{11}^{0}(p)=G_{F}^{0}(p)+\Gamma^{0}(p) \\
& G_{22}^{0}(p)=G_{F}^{0}(p)^{*}+\Gamma^{0}(p) \\
& G_{12}^{0}(p)=D_{F}^{0}(p)+\Gamma^{0}(p) \\
& G_{21}^{0}(p)=D_{F}^{0}(-p)+\Gamma^{0}(p)
\end{aligned}
$$

where

$$
\begin{aligned}
G_{F}^{0}(p) & =\frac{i}{p^{2}-m^{2}+i \epsilon} \\
D_{F}^{0}(p) & =\theta\left(p^{0}\right) 2 \pi \delta\left(p^{2}-m^{2}\right)
\end{aligned}
$$

are the vacuum Feynman propagator and the vacuum Cutkosky propagator, respectively. To compute the Wightman self-energy using this set of propagators, one must use the following Feynman rules [22]:

1. Draw all cut diagrams where the cut separates the two external vertices. Label $\Pi^{>}(k)$ all diagrams where the external momentum $k$ enters the unshaded ('1') region. Label $\Pi^{<}(k)$ all diagrams where $k$ enters the shaded ('2') region.

2. Use the usual Feynman rules for the unshaded side assigning $G_{11}^{0}(p)$ to the uncut lines. For the shaded side, use the conjugate Feynman rules assigning $G_{22}^{0}(p)$ to the uncut lines.

3. If the momentum of a cut line crosses from the unshaded to the shaded region, assign $G_{12}^{0}(p)$. If the momentum of a cut line crosses from the shaded to the unshaded region, assign $G_{21}^{0}(p)$.

4. Divide by the appropriate symmetry factor.

To see an explicit example employing the above rules, see Appendix A.

Note that each propagator can be separated into a zero temperature part and a finite temperature part. Furthermore, the thermal phase space factor

$$
\Gamma^{0}(p)=n\left(\left|p^{0}\right|\right) 2 \pi \delta\left(p^{2}-m^{2}\right)
$$

\footnotetext{
${ }^{1}$ In this paper, we concentrate on scalar theories because we want to reproduce the results of [16]. However, the multiple scattering expansion is general and does not rely on any specific Lagrangian.
} 
is common to all $G_{i j}^{0}$ and the zero-temperature part of the propagators are exactly those of the vacuum Cutkosky rules [30]. Therefore any diagram in this approach can be naturally divided into a thermal part $\left(\Gamma^{0}(p)\right)$ and a zero-temperature part.

Using the above two properties of the finite temperature propagators, it is possible to expand any diagram as a power series in the number of thermal phase space factor. In this expansion, each coefficient of the expansion is a zero temperature scattering diagram [22].

At a first glance, this looks like a fairly complicated expansion. First, for any given topology of a diagram, one needs to consider all relevant thermal cuts (i.e. sum of all different combinations of 1 and 2 vertices) [29]. Then each of these thermal cut diagrams must be expanded again according to the number of $\Gamma^{0}$ 's. This expansion becomes manageable only if one can show that the coefficients of the same $\Gamma^{0}$ factors have a simple meaning in the context of the vacuum theory.

That these coefficients cannot be the usual Feynman diagrams is evident from the appearance of the cutting rule propagators in Eqs.(14][17). Hence, the coefficients must correspond to the vacuum Cutkosky (cut) diagrams. In vacuum theory, Cutkosky diagrams are needed when one needs to work with the unitarity condition

$$
2 \operatorname{Im} \mathcal{T}=\mathcal{T}^{\dagger} \mathcal{T}
$$

where $i \mathcal{T}=\mathcal{S}-1$ is the transition operator. Therefore, roughly speaking

(i) The $G_{F}^{0}$ part of $G_{11}^{0}$ propagators form Feynman diagrams that correspond to the matrix elements of $\mathcal{T}$, whereas the $G_{F}^{0 *}$ part of $G_{22}^{0}$ propagators form Feynman diagrams that correspond to the matrix elements of $\mathcal{T}^{\dagger}$.

(ii) The $D_{F}^{0}$ part of $G_{12}^{0}$ and $G_{21}^{0}$ propagators provide the intermediate states connecting $\mathcal{T}^{\dagger}$ and $\mathcal{T}$ matrix elements.

(iii) The thermal part $\Gamma^{0}$ represents the bath of on-shell particles in the medium that participate in the collision process described by the matrix elements of $\mathcal{T}$ and $\mathcal{T}^{\dagger}$.

In Ref.[22], it was argued that following this reasoning yields

$$
\begin{aligned}
\Pi^{>}(k)=\sum_{m=0}^{\infty} \sum_{n=0}^{\infty} \frac{1}{m ! n !}\left(\int\right. & \left.\prod_{i=1}^{m} \frac{d^{3} l_{i}}{(2 \pi)^{3} 2 E_{l_{i}}} n\left(E_{l_{i}}\right)\right)\left(\int \prod_{j=1}^{n} \frac{d^{3} p_{j}}{(2 \pi)^{3} 2 E_{p_{j}}}\right) \\
& \times\left.\left\langle k,\left\{l_{i}\right\}_{m}\left|\mathcal{T}_{k}^{\dagger}\right|\left\{p_{j}\right\}_{n}\right\rangle\left\langle\left\{p_{j}\right\}_{n}\left|\mathcal{T}_{k}\right| k,\left\{l_{i}\right\}_{m}\right\rangle\right|_{\text {conn. }}
\end{aligned}
$$

The subscripts $m$ and $n$ in $\left\{l_{i}\right\}_{m}$ and $\left\{p_{j}\right\}_{n}$ indicate that there are $m l_{i}$ 's and $n p_{j}$ 's in each set. In here and the following, we use the label $k$ exclusively for the external momentum entering the unshaded side of the self-energy diagram. The subscript $k$ on the operator $\mathcal{T}_{k}$ means 


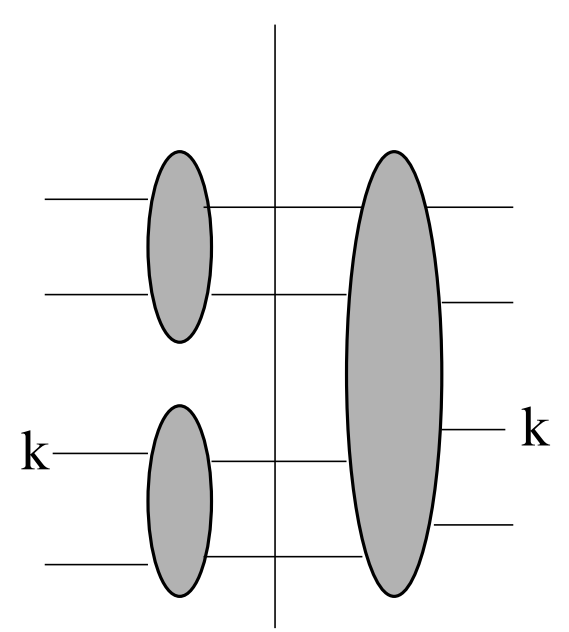

Included

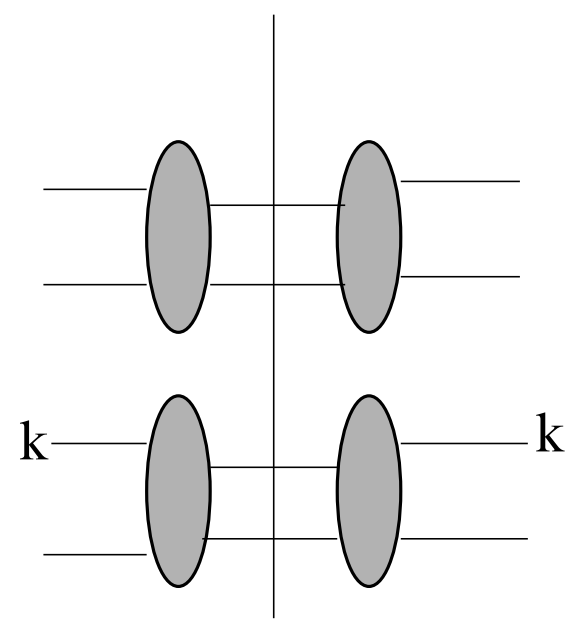

Not included

FIG. 1: An illustration of diagrams that are included and not included in $\left.\left\langle k,\left\{l_{i}\right\}_{m}\left|\mathcal{T}_{k}^{\dagger}\right|\left\{p_{j}\right\}_{n}\right\rangle\left\langle\left\{p_{j}\right\}_{n}\left|\mathcal{T}_{k}\right| k,\left\{l_{i}\right\}_{m}\right\rangle\right|_{\text {conn }}$

that the external momentum always interacts with the other momentum states. Note that we do not write $\left|\left\langle\left\{p_{j}\right\}_{n}\left|\mathcal{T}_{k}\right| k,\left\{l_{i}\right\}_{m}\right\rangle\right|^{2}$ but leave $\left\langle k,\left\{l_{i}\right\}_{m}\left|\mathcal{T}_{k}^{\dagger}\right|\left\{p_{j}\right\}_{n}\right\rangle$ and $\left\langle\left\{p_{j}\right\}_{n}\left|\mathcal{T}_{k}\right| k,\left\{l_{i}\right\}_{m}\right\rangle$ explicitly separated; this is to express that even if the momenta $\left\{p_{j}\right\}$ can be interconnected in all possible ways, some diagrams needed to make a squared matrix element are missing. The subscript "conn." indicates that the joining of $\mathcal{T}_{k}^{\dagger}$ and $\mathcal{T}_{k}$ does not give disconnected pieces, even if by itself $\mathcal{T}_{k}$ contains both clusters and spectators (see Figs. 1] and 2). A cluster is a connected amplitude for a certain subset of initial and final momentum states that is completely disconnected from the other amplitudes. Note here that the denomination "initial" and "final" when applied to states sandwiching $\mathcal{T}_{k}$ is slightly different from states sandwiching the fully connected scattering amplitude (see Fig. 3 and the discussion around Eqs.(32)-(33)). A spectator is a state that does not interact with other states (see Fig. 22). For future reference, we also define "fully connected" as the sum of all diagrams where all momentum states are connected together when joining two amplitudes (see Fig. 2 for an illustration of this).

\section{B. Medium Related Complications}

To turn the collision term of the Kadanoff-Baym equation into the usual Boltzmann equation collision term, we must determine under what conditions Eq.(22) can be approximated 


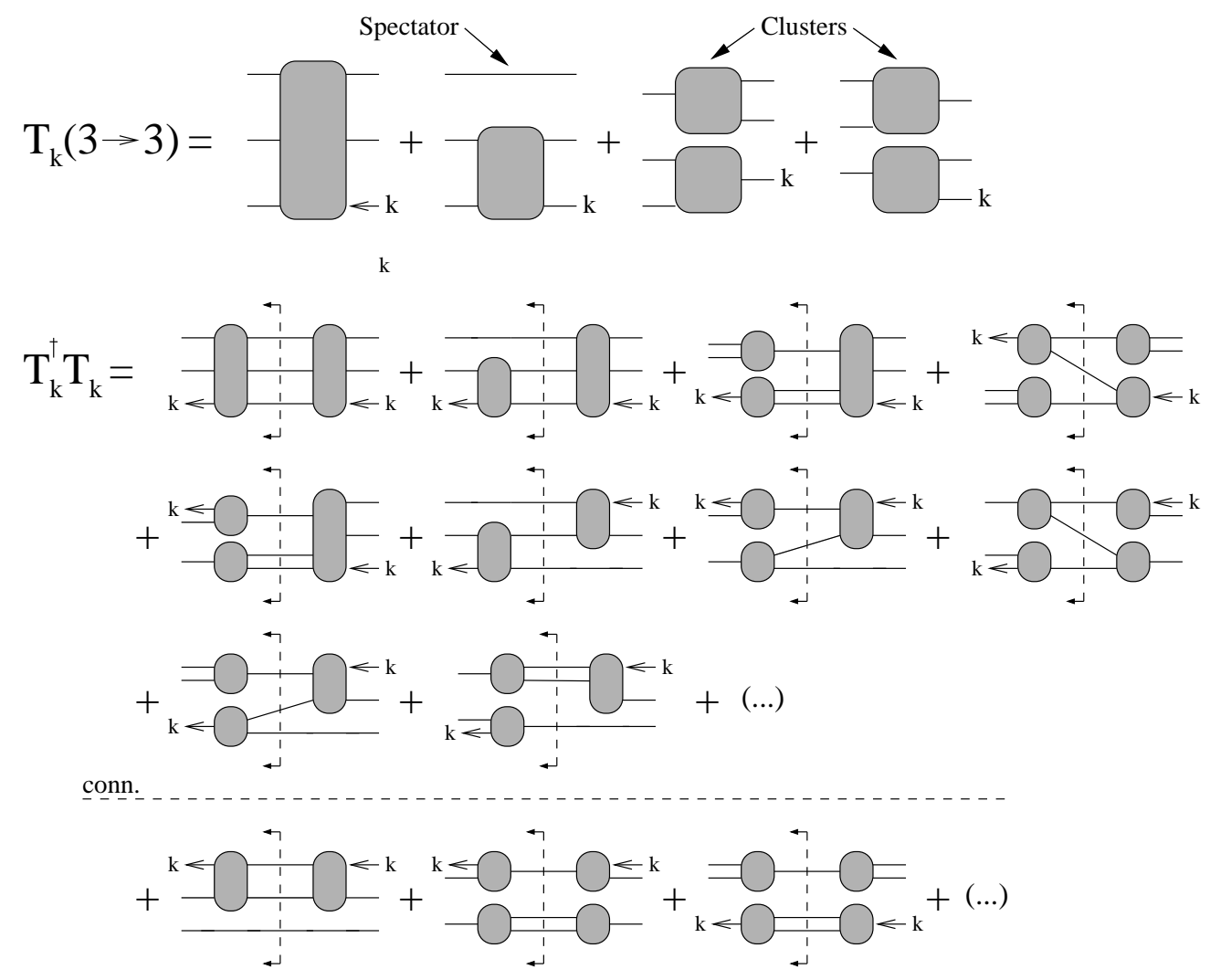

FIG. 2: An example of a $3 \rightarrow 3$ process included in the multiple scattering expansion $\left.\left\langle k,\left\{l_{i}\right\}_{2}\left|\mathcal{T}_{k}^{\dagger}\right|\left\{p_{j}\right\}_{3}\right\rangle\left\langle\left\{p_{j}\right\}_{3}\left|\mathcal{T}_{k}\right| k,\left\{l_{i}\right\}_{2}\right\rangle\right|_{\text {conn. }}$. The lines cut by the dashed lines are final states and the arrows indicate the complex conjugated side. The blobs represent connected amplitudes. The $\left\{p_{j}\right\}_{3}$ are the momenta of the final states while the $\left\{l_{i}\right\}_{2}$ are the initial states weighted by thermal factors. The external momentum $k$ is always interacting. $\mathcal{T}_{k}$ contains both clusters and spectators. The expression $\mathcal{T}_{k}^{\dagger} \mathcal{T}_{k}$ contains all the possible connections between each other. The (...) represents diagrams similar to the ones already shown but with all momenta and the shading reversed. The subscript conn. selects all the diagrams above the dashed line. The first diagram of $\mathcal{T}_{k}^{\dagger} \mathcal{T}_{k}$ is the only fully connected diagram.

by

$$
\begin{gathered}
\Pi_{\text {Boltz }}^{>}(k)=\sum_{s=0}^{\infty} \sum_{n=0}^{\infty} \frac{1}{n ! s !}\left(\int \prod_{i=1}^{n} \frac{d^{3} l_{i}}{(2 \pi)^{3} 2 E_{i}} n\left(E_{i}\right)\right)\left(\int \prod_{j=1}^{s} \frac{d^{3} p_{j}}{(2 \pi)^{3} 2 E_{j}}\left[1+n\left(E_{j}\right)\right]\right) \\
\times\left|\left\langle\left\{p_{j}\right\}_{s}|\mathcal{M}| k,\left\{l_{i}\right\}_{n}\right\rangle\right|^{2}
\end{gathered}
$$

where $\left|\left\langle\left\{p_{j}\right\}_{s}|\mathcal{M}| k,\left\{l_{i}\right\}_{n}\right\rangle\right|^{2}$ is the square of the fully connected scattering matrix element between the initial momenta $k$ and $\left\{l_{i}\right\}_{n}$ and the final momenta $\left\{p_{j}\right\}_{s}$. This is the main goal of our paper. Note that $\mathcal{M}$ does not carry the subscript $k$ since it is fully connected. 
The presence of the medium, however, complicates this task. Due to the processes occuring within the medium, the $\left\langle\left\{p_{j}\right\}_{n}\left|\mathcal{T}_{k}\right|\left\{l_{i}\right\}_{m}\right\rangle$ piece appearing in Eq.(22) does not have to be composed of only the fully connected diagrams. Some of the initial momenta $\left\{l_{i}\right\}_{m}$ and the final momenta $\left\{p_{j}\right\}_{n}$ can interact among themselves without interacting with the main piece where $k$ interacts. This is illustrated in Fig. 团and Fig. 2] Figure 1 also illustrates why the factor

$$
\left.\mathcal{K}_{T}\left(k,\left\{l_{i}\right\}_{m},\left\{p_{j}\right\}_{n}\right) \equiv\left\langle k,\left\{l_{i}\right\}_{m}\left|\mathcal{T}_{k}^{\dagger}\right|\left\{p_{j}\right\}_{n}\right\rangle\left\langle\left\{p_{j}\right\}_{n}\left|\mathcal{T}_{k}\right| k,\left\{l_{i}\right\}_{m}\right\rangle\right|_{\text {conn }}
$$

cannot be readily interpreted as the square of a fully connected scattering matrix element. The right hand side diagram in Fig. 1 must appear in the square of the $\mathcal{T}$ matrix element, yet it is missing in $\mathcal{K}_{T}$ because these types of diagrams do not appear naturally in the self-energy calculation. This fact is taken into account by the "conn." prescription.

The occurence of 'spectators' and 'clusters' in a $\mathcal{T}$ matrix element is well known (for instance, see 31]). In vacuum theory, these disconnected pieces do not contribute to the self-energy calculation because they represent off-shell vacuum fluctuations which are independent of the scattering process. At finite temperature, however, these in-medium processes can no longer be neglected. This is because the disconnected pieces can now represent real processes occuring between on-shell particles in the medium. Furthermore, quantum interference can occur between these $k$-independent in-medium processes and the process where

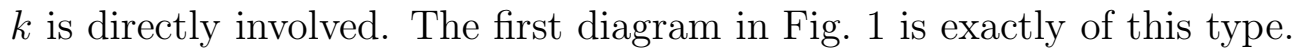

Another complication is the fact that no momentum state is stable at finite temperature since, within a mean free path, the momentum of any particle will likely change. This implies that there are no real asymptotic states at finite temperature [32, 33]. Therefore, one must always start with resummed propagators and make an appropriate quasi-particle approximation.

The use of resummed propagators is also essential to deal with so-called "pinch" singularities. When computing diagrams in finite temperature quantum field theory, one must be careful with self-energy insertions since the resulting product of contour propagators can give rise to ill defined products of delta functions. Fortunately, for equilibrium systems, these singularities cancel due to the KMS condition [22, 32, 35]. However, for outof-equilibrium systems in which the KMS condition is not satisfied, those singularities do not cancel 34, 35, 36, 37, 38] at any finite order in perturbation theory. But as pointed out in 36, 37, 38], an out-of-equilibrium system is integrated only over a finite volume; thus, products of delta functions do not diverge in physical situations. As discussed in Refs. 35, 36, 37, 38], the way around this difficulty is to use resummed propagators, where no self-energy insertions (and thus no pinch singularities) appear. To show that Eq.(23) is indeed a good approximation of Eq.(22) and to deal with pinch singularities correctly, it is 
thus essential to use resummed propagators.

\section{Resummed Perturbation Theory and the Skeleton Expansion}

The spectral density for a stable particle in vacuum has two separate pieces, an on-shell $\delta$-function piece and an off-shell continuum piece. Stability requires that these two pieces are well separated. In a medium, however, on-shell states are not stable since elastic collisions can easily change their momenta. This implies that the zero temperature part and the in-medium part may not separate cleanly.

Unless the external particles are either stable or we assume that the interactions are turned off at $t= \pm \infty$, the scattering matrix elements between on-shell particles are not well defined. Technically speaking, the validity of the LSZ formalism depends on the fact that we can cleanly amputate the external legs of a Feynman diagram. That is, the selfenergy insertions along any external leg result only in renormalization of the mass and the wavefunction. At finite temperature, this can no longer be cleanly achieved because the self-energy has a non-zero imaginary part for any momentum. In other words, both the on-shell piece and the continuum piece in the spectral density broaden so that there is no longer a gap between them. Nevertheless, if the interaction strength is weak and the medium is weakly inhomogeneous, the on-shell piece and the continuum piece should be separated enough so that we can use a 'quasi-particle' approximation. We will do so in a short while. Here, we first discuss the skeleton expansion whose result is needed later.

To address the self-energy problem in a systematic way, consider the resummed perturbation theory where the propagators are fully resummed. In such a theory, the propagators in Eqs.(14,17]) become:

$$
\begin{aligned}
& G_{11}(p)=G_{F}(p)+\Gamma(p) \\
& G_{22}(p)=G_{F}^{*}(p)+\Gamma(p) \\
& G_{12}(p)=\theta\left(p^{0}\right) \rho(p)+\Gamma(p) \\
& G_{21}(p)=-\theta\left(-p^{0}\right) \rho(p)+\Gamma(p)
\end{aligned}
$$

where

$$
G_{F}(p)=\frac{i}{p^{2}-m^{2}+\Pi_{R}(p)+i\left|\Pi_{I}(p)\right|}
$$

is the resummed analogue of the Feynman propagator and

$$
\rho(p)=\frac{2 \Pi_{I}(p)}{\left|p^{2}-m^{2}+\Pi_{R}(p)+i \Pi_{I}(p)\right|^{2}}
$$


is the resummed spectral density. The thermal factor is given by:

$$
\Gamma(p)=n\left(\left|p^{0}\right|\right) \operatorname{sgn}\left(p^{0}\right) \rho(p)
$$

In these expressions, $\Pi_{R}(p)$ and $\Pi_{I}(p)$ are the real and imaginary parts of the retarded self-energy and $\operatorname{sgn}(x)$ denotes the sign of $x$.

The original multiple scattering expansion of the self-energy (c.f. Eq.(22) ) is based on an expansion in terms of thermal factors. As mentioned earlier, we need to use resummed propagators in order to deal with pinch singularities correctly. Because of this fact, Eq.(22) is not useful in a practical sense. It must be revisited using resummed perturbation theory. Moreover, to reproduce "Boltzmann" Wightman's functions (c.f. Eq.(23)), it is convenient to modify the original expansion so that thermally weighted initial and final states appear more naturally. Thus, we follow a reasoning analogous to the one that led to Eq.(22) but using resummed propagators and organizing the perturbation series differently. In order to do that, we rewrite the propagators $G_{12}$ and $G_{21}$ as

$$
\begin{aligned}
& G_{12}(p)=\theta\left(p^{0}\right)\left[1+n\left(\left|p^{0}\right|\right)\right] \rho_{+}(p)+\theta\left(-p^{0}\right) n\left(\left|p^{0}\right|\right) \rho_{+}(p) \\
& G_{21}(p)=\theta\left(-p^{0}\right)\left[1+n\left(\left|p^{0}\right|\right)\right] \rho_{+}(p)+\theta\left(p^{0}\right) n\left(\left|p^{0}\right|\right) \rho_{+}(p)
\end{aligned}
$$

with

$$
\rho_{+}(p)=\operatorname{sgn}\left(p^{0}\right) \rho(p)=\rho\left(\left|p^{0}\right|, \mathbf{p}\right)
$$

The $\left[1+n\left(\left|p^{0}\right|\right)\right] \rho_{+}(p)$ factor in $G_{12}$ and $G_{21}$ is naturally associated with a final state momentum in any given scattering process, and the $n\left(\left|p^{0}\right|\right) \rho_{+}(p)$ factor in $G_{12}$ and $G_{21}$ is associated with an initial state momentum in any given scattering process. The idea is to expand the full self-energy using the propagators (32)-(33); see Fig. 3 for an illustration of this procedure. Note that since we use resummed propagators, only skeleton diagrams appear in the self-energy. As usual, skeleton diagrams are defined as diagrams without any self-energy insertions. The result of this procedure can be written in a very suggestive form:

$$
\begin{aligned}
\Pi^{>}(k)=\sum_{n=0}^{\infty} \sum_{s=0}^{\infty} \frac{1}{n ! s !} & \left(\int \prod_{i=1}^{n} d \Gamma_{-}\left(l_{i}\right)\right)\left(\int \prod_{j=1}^{s} d \Gamma_{+}\left(p_{j}\right)\right) \\
& \times\left.\left\langle k,\left\{l_{i}\right\}_{n}\left|\mathcal{A}_{k}^{\dagger}\right|\left\{p_{j}\right\}_{s}\right\rangle\left\langle\left\{p_{j}\right\}_{s}\left|\mathcal{A}_{k}\right| k,\left\{l_{i}\right\}_{n}\right\rangle\right|_{\text {skeleton }}
\end{aligned}
$$

where the resummed phase space factors are given by

$$
\begin{aligned}
& d \Gamma_{-}(p)=\frac{d^{4} p}{(2 \pi)^{4}} \theta\left(p^{0}\right) \rho_{+}(p) n\left(\left|p^{0}\right|\right) \\
& d \Gamma_{+}(p)=\frac{d^{4} p}{(2 \pi)^{4}} \theta\left(p^{0}\right) \rho_{+}(p)\left[1+n\left(\left|p^{0}\right|\right)\right]
\end{aligned}
$$



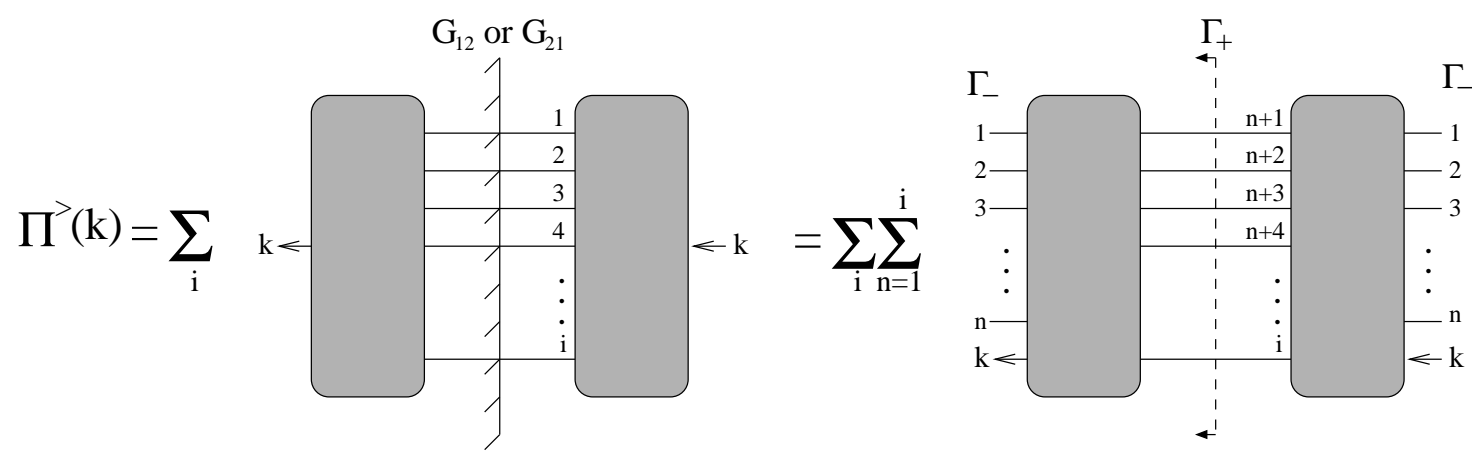

FIG. 3: The first diagram represents the sum over all skeleton diagrams that have $i$ cut lines. The blob on the shaded side is made of $G_{22}(p)$ 's while the blob on the unshaded side is made of $G_{11}(p)$ 's. Cut lines are associated with the cut propagators $G_{12}(p)$ and $G_{21}(p)$. The second diagram is obtained from the first by expanding the cut propagators using Eq. (38) and by changing the variable so that $\theta\left(-p^{0}\right) \rightarrow \theta\left(p^{0}\right)$. Thus, all the states weighted by $d \Gamma^{+}$becomes final states while the states weighted by $d \Gamma^{-}$becomes initial states. The lines cut by the dashed line are final states. The arrows indicate the complex conjugated side. We neglected symmetry factors for simplicity.

with the relation (true for rotationally invariant systems)

$$
d \Gamma_{+}(p)+d \Gamma_{-}(-p)=\frac{d^{4} p}{(2 \pi)^{4}} G_{12}(p)
$$

Here $\left\langle\left\{p_{j}\right\}_{s}\left|\mathcal{A}_{k}\right| k,\left\{l_{i}\right\}_{n}\right\rangle$ is purely made of $G_{11}$ and represents the sum of all amputated skeleton diagrams ${ }^{2}$ between a set of initial momenta $\left\{l_{i}\right\}_{n}$ and a set of final momenta $\left\{p_{j}\right\}_{s}$. The factor $\left\langle k,\left\{l_{i}\right\}_{n}\left|\mathcal{A}_{k}^{\dagger}\right|\left\{p_{j}\right\}_{s}\right\rangle$ has the same interpretation and is purely made of $G_{22}$; however, it must be emphasized that

$$
\mathcal{K}_{A}\left(k,\left\{l_{i}\right\}_{n},\left\{p_{j}\right\}_{s}\right)=\left.\left\langle k,\left\{l_{i}\right\}_{n}\left|\mathcal{A}_{k}^{\dagger}\right|\left\{p_{j}\right\}_{s}\right\rangle\left\langle\left\{p_{j}\right\}_{s}\left|\mathcal{A}_{k}\right| k,\left\{l_{i}\right\}_{n}\right\rangle\right|_{\text {skeleton }}
$$

is not the fully connected matrix element squared that appears in the Boltzmann collision term. This will be examined further in the next section.

\section{Approximations}

We know that in order to interpret the right hand side of the Kadanoff-Baym equation (c.f. Eq.(13) ) as the collision integral in the Boltzmann equation, the self-energy $\Pi^{>}$(which appears in the loss term) should have the form shown in Eq.(23). Comparing Eq.(23) with Eq.(35) one can see the following differences:

\footnotetext{
${ }^{2}$ Note that Eq.(35) contains no spectator, but still includes clusters. We deal with the latter in Sect. IIID
} 
(i) The momentum integrals in Eq.(23) are over on-shell 3-momenta whereas the momentum integrals in Eq.(35) are over 4-momenta.

(ii) There are some extra terms in Eq.(39). First, $\mathcal{K}_{A}$ is made of the thermal propagators $G_{11}(q)$ and $G_{22}(q)$ while $\left\langle\left\{p_{j}\right\}|\mathcal{M}| k,\left\{l_{i}\right\}\right\rangle$ contains only the vacuum ones $\left(G_{F}^{0}(q)\right.$ and $\left.G_{F}^{0 *}(q)\right)$. Also, $\mathcal{K}_{A}$ is made of connected diagrams that contains clusters (but no spectators), while $\left\langle\left\{p_{j}\right\}|\mathcal{M}| k,\left\{l_{i}\right\}\right\rangle$ contains only the fully connected diagrams (see Fig. 2 for the difference between connected and fully connected).

(iii) There are some missing terms in Eq.(39). The terms of the form $G_{F}^{*}(q) G_{F}(q)$ (see Fig. [5] and example in Appendix (A) are not part of the skeleton expansion in Eq.(35), by definition. These terms were present in the original multiple scattering expansion Eq.(22), so they appear to be lost in the skeleton expansion of resummed propagators. They are actually included in the resummed phase space factor.

Since the Boltzmann equation is only a classical approximation to the full KadanoffBaym equation, one should not expect that $\Pi_{\text {Boltz. }}^{>}(k)$ in Eq.(23) is exactly the same as $\Pi^{>}(k)$ in Eq.(35). Rather, the question to ask is, "In what sense is Eq.(23) contained in Eq.(35)?" Physically, the Boltzmann equation should be a good approximation in the weakly-interacting dilute-gas limit. Hence, it should be no surprise that the answer to the above question is provided by a quasi-particle ansatz.

First consider point (i). The connection between the 4-momentum and the on-shell 3momentum is provided by the following quasi-particle approximation. In a medium, no momentum state can be absolutely stable at finite temperature. However, the Boltzmann equation is an equation for the on-shell particle density. This means that it is valid only when the mean free path is much longer than the range of the interaction, which occurs in weakly interacting systems (i.e. the usual approximations of transport theory, see Sect. II] and [16]). This leads to the following condition for the quasi-particle approximation

$$
m^{2}-\Pi_{R} \gg\left|\Pi_{I}^{T}\right|
$$

where $\Pi_{I}^{T}$ means the imaginary part of the thermal self-energy. If Eq.(40) holds, the onshell part of the spectral function is well separated from the continuum part (see Fig. 4 and 14, 41]) and it becomes possible to make the following quasi-particle approximation

$$
\begin{aligned}
\rho_{+}(k) & =2 \Pi_{I}\left(\left|k_{0}\right|, k\right) G_{F}(k) G_{F}^{*}(k) \\
\rightarrow \rho_{+}^{\text {quasi }}(k) & =2 \pi \delta\left(k^{2}-m_{T}^{2}\right)+2 \Pi_{I}(k) G_{V}(k) G_{V}^{*}(k) \theta\left(k^{2} \neq m_{T}^{2}\right)
\end{aligned}
$$




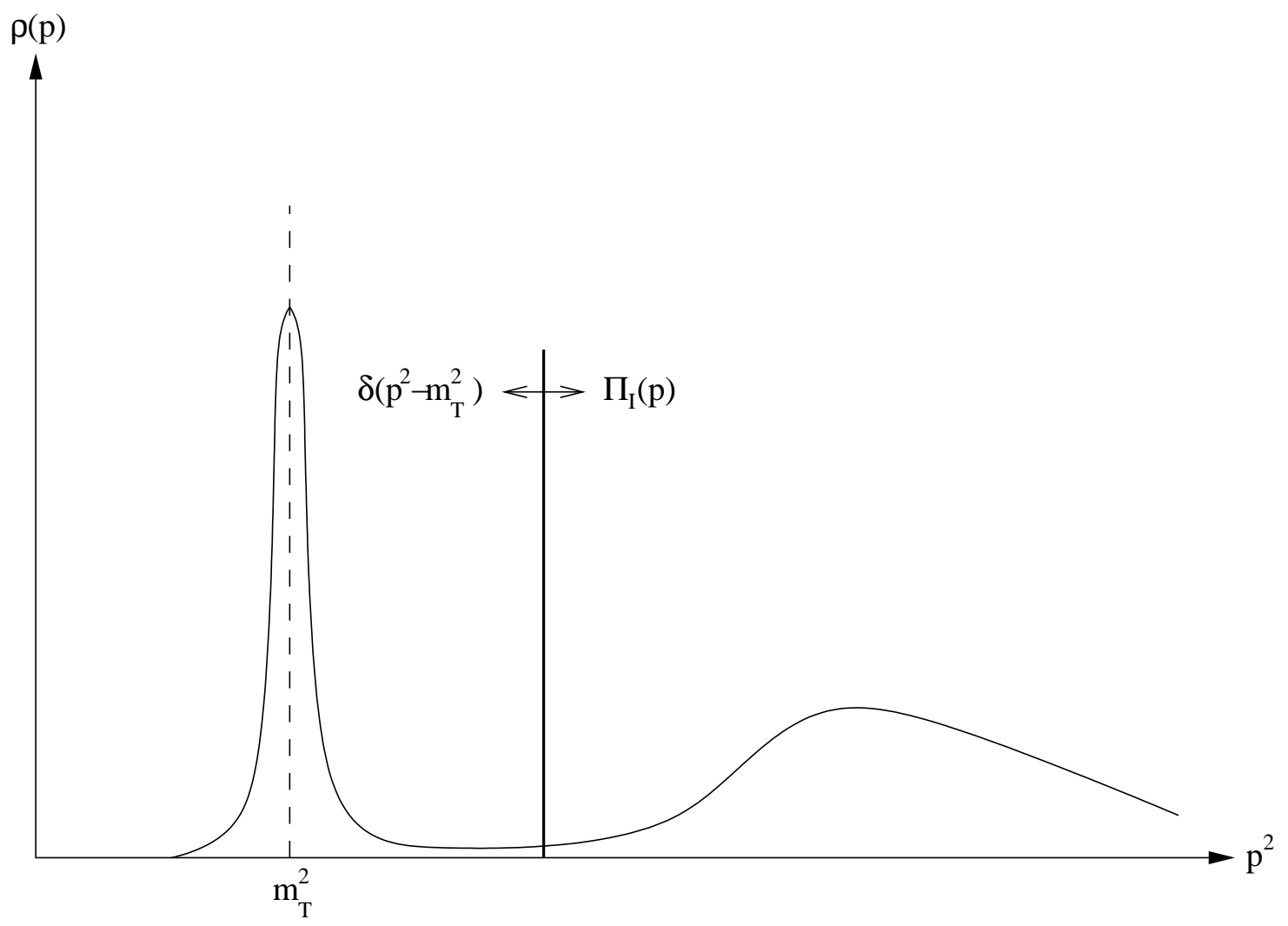

FIG. 4: Qualitative depiction of the spectral density. In the quasi-particle approximation, the left-hand side is approximated by $\delta\left(p^{2}-m_{T}^{2}\right)$ while the right-hand side is given by $\Pi_{I}(p)$.

where the $\theta$-function ensures that we do not double-count the on-shell contribution. Here $m_{T}^{2}=m^{2}-\Pi_{R}$ is the thermal mass and we defined

$$
G_{V}(p)=\frac{i}{p^{2}-m_{T}^{2}+i \Pi_{I}^{V}(p)}
$$

with $\Pi_{I}^{V}$ denoting the imaginary part of the vacuum self-energy. Equations (411)-(42) constitute our quasi-particle approximation.

Point (ii) is about clusters in $\mathcal{K}_{A}$. The diagrams that contain clusters represent interference between the processes that are occuring within the medium and the scattering process in which the original external momentum $k$ is involved. For instance, see the diagram on the left hand side of Fig. 1. As such, it is not possible to interpret these terms as a matrix element squared because terms representing independent scattering processes (as shown on the right hand side of Fig. (1) are missing. It is conceivable that one may introduce these missing terms via a multiplicative constant analogous to an effective potential. But in this work, we need to ignore these interference terms and note that this is a defect of the Boltzmann 
equation approach. We simply replace $\mathcal{K}_{A}$ with

$$
\left.\mathcal{K}_{A}\left(k,\left\{l_{i}\right\}_{n},\left\{p_{j}\right\}_{s}\right) \rightarrow\left\langle k,\left\{l_{i}\right\}_{n}\left|\mathcal{M}^{\dagger}\right|\left\{p_{j}\right\}_{s}\right\rangle\left\langle\left\{p_{j}\right\}_{s}|\mathcal{M}| k,\left\{l_{i}\right\}_{n}\right\rangle\right|_{\text {skeleton }}
$$

where $\left\langle\left\{p_{j}\right\}_{s}|\mathcal{M}| k,\left\{l_{i}\right\}_{n}\right\rangle$ now consists solely of fully connected networks of $G_{V}$ 's (c.f. Eq.(42)). Note that the restriction that no self-energy insertion appear still applies. Note also that ignoring these independent scattering processes (equivalent to neglecting the thermal factors $\Gamma(p)$ in Eqs.(25)-(26) ) would break gauge invariance in a gauge theory. As

is well-known from the Ward identities for Hard Thermal Loops (HTLs) 39, 40], both propagators and vertices must be HTL resummed to preserve gauge invariance. In our case, throwing away these independent scattering processes would prevent getting the necessary thermal corrections to the vertices needed for preserving gauge invariance. We come back to this point and possible solutions in Sect. $\nabla$

Point (iii) is concerned with the problem of generating all the missing diagrams in our skeleton expansion so that we recover full matrix elements squared. This is a non-trivial problem and we devote the next section to its solution. In order to make progress, we need to use the following relationships:

$$
\begin{array}{r}
\theta\left(p^{0}\right) n\left(\left|p^{0}\right|\right) 2 \Pi_{I}(p)=\theta\left(p^{0}\right) \Pi^{<}(p) \\
\theta\left(p^{0}\right)\left[1+n\left(\left|p^{0}\right|\right)\right] 2 \Pi_{I}(p)=\theta\left(p^{0}\right) \Pi^{>}(p)
\end{array}
$$

Equations (44)-(45) are a rewriting of the usual KMS condition $n\left(p^{0}\right) \Pi^{>}(p)=(1+$ $\left.n\left(p^{0}\right)\right) \Pi^{<}(p)$. This relation is only valid in equilibrium and makes the collision term of the Kadanoff-Baym equation vanish identically. However, in an nonequilibrium system, the KMS condition is not satisfied and should not be used. We argue here that it is still consistent to use Eqs.(44)-(45) even if we are in a nonequilibrium setting as long as we only use it in Eq.(46) for the computation of $\Pi^{>}(p)$ and $\Pi^{<}(p)$. The key observation is that $\Pi^{>}(p)$ and $\Pi^{<}(p)$ are proportional to each other up to gradient terms: this is the essence of the Kadanoff-Baym equation (11). The effect of these gradient terms would be to add higher order gradient terms in our computation of $\Pi^{>}(p)$ and $\Pi^{<}(p)$. These higher order terms can be neglected, since the Kadanoff-Baym equation is obtained from an expansion to lowest order in the gradients. In this sense, Eqs.(44)-(45) are approximate expressions that are consistent with the the Kadanoff-Baym equation.

\section{COLLISION TERM DERIVATION}

As explained in Sect. ПIID, we need to show how the missing terms in the squared scattering matrix elements are generated. With the approximations presented in the last 
section, Eq.(35) becomes

$$
\begin{aligned}
\Pi^{>}(k)= & \sum_{n=0}^{\infty} \sum_{s=0}^{\infty} \frac{1}{s ! n !} \int \prod_{k=1}^{n} \frac{d^{4} l_{k}}{(2 \pi)^{4}} \theta\left(l_{k}^{0}\right)\left[n\left(\left|l_{k}^{0}\right|\right) 2 \pi \delta\left(l_{k}^{2}-m_{T}^{2}\right)+G_{V}^{*}\left(l_{k}\right) \Pi^{<}\left(l_{k}\right) G_{V}\left(l_{k}\right) \theta\left(k^{2} \neq m_{T}^{2}\right)\right] \\
& \times \int \prod_{j=1}^{s} \frac{d^{4} p_{j}}{(2 \pi)^{4}} \theta\left(p_{j}^{0}\right)\left[\left[1+n\left(\left|p_{j}^{0}\right|\right)\right] 2 \pi \delta\left(p_{j}^{2}-m_{T}^{2}\right)+G_{V}\left(p_{j}\right) \Pi^{>}\left(p_{j}\right) G_{V}^{*}\left(p_{j}\right) \theta\left(k^{2} \neq m_{T}^{2}\right)\right] \\
& \times\left.\left\langle k,\left\{l_{k}\right\}_{n}\left|\mathcal{M}^{\dagger}\right|\left\{p_{j}\right\}_{s}\right\rangle\left\langle\left\{p_{j}\right\}_{s}|\mathcal{M}| k,\left\{l_{k}\right\}_{n}\right\rangle\right|_{\text {skeleton }}
\end{aligned}
$$

with a similar expression for $\Pi^{<}(k)$. This equation defines a self-consistent equation for $\Pi^{>}$ and $\Pi^{<}$. Our problem is now reduced to showing that the Boltzmann ansatz, Eq.(23), is a solution of Eq.(46).

Qualitatively, we can argue as follows: the scattering matrix element $\left\langle\left\{p_{j}\right\}|\mathcal{M}| k,\left\{l_{k}\right\}\right\rangle$ is now made of zero temperature Feynman propagators $G_{V}(p)$. However, we cannot yet say that

$$
\mathcal{K}_{M}=\left.\left\langle k,\left\{l_{k}\right\}_{n}\left|\mathcal{M}^{\dagger}\right|\left\{p_{j}\right\}_{s}\right\rangle\left\langle\left\{p_{j}\right\}_{s}|\mathcal{M}| k,\left\{l_{k}\right\}_{n}\right\rangle\right|_{\text {skeleton }}
$$

equals $\left|\left\langle\left\{p_{j}\right\}_{s}|\mathcal{M}| k,\left\{l_{k}\right\}_{n}\right\rangle\right|^{2}$. This is again because diagrams in the skeleton expansion cannot include self-energy insertions and hence do not contain all Feynman diagrams contributing to $\left|\left\langle\left\{p_{j}\right\}_{s}|\mathcal{M}| k,\left\{l_{k}\right\}_{n}\right\rangle\right|^{2}$. We should emphasize here that the matrix element $\left\langle\left\{p_{j}\right\}_{s}|\mathcal{M}| k,\left\{l_{k}\right\}_{n}\right\rangle$ itself does contain all necessary diagrams. It is the joining of the final state momenta $\left\{p_{j}\right\}_{s}$ that makes the distinction between $\left|\left\langle\left\{p_{j}\right\}_{s}|\mathcal{M}| k,\left\{l_{k}\right\}_{n}\right\rangle\right|^{2}$ and $\mathcal{K}_{M}$. This is illustrated in Fig. 5 for the part of $\Pi^{>}$which involves 4 particle (2-2 or 1-3) processes. The same figure also illustrates that the missing diagrams are the ones with self-energy insertions.

Since the right hand side of Eq.(46) includes $\Pi^{>}$and $\Pi^{<}$, this equation must be solved iteratively; hence, the missing diagrams must come from this iteration procedure. In other words, diagrams missing in the skeleton sum $\mathcal{K}_{M}$ should all be generated by the lower order (in the number of legs) skeleton diagrams by trading some on-shell $\delta$-functions with $G_{V}^{*} \Pi^{>},<G_{V}$ combinations. For example, the diagram labelled (e) in Fig. 5 is generated by one iteration as shown in Fig. 6. Fig. 7 contains a more elaborate example. Hence, one can at least see that Eq.(23) and Eq.(46) contain the same set of diagrams. If the symmetry factors all work out, then we have shown that the two expressions are equivalent. An example of this procedure involving 2 to 2 scattering processes is presented in Appendix A.

Formally, what we need to show is that Eq.(23) is the iterated solution of Eq.(46). To do so, we define

$$
\begin{aligned}
& d \Gamma_{-}^{0}(p)=\frac{d^{4} p}{(2 \pi)^{4}} \theta\left(p^{0}\right) n\left(\left|p^{0}\right|\right) 2 \pi \delta\left(p^{2}-m_{T}^{2}\right) \\
& d \Gamma_{+}^{0}(p)=\frac{d^{4} p}{(2 \pi)^{4}} \theta\left(p^{0}\right)\left[1+n\left(\left|p^{0}\right|\right)\right] 2 \pi \delta\left(p^{2}-m_{T}^{2}\right)
\end{aligned}
$$




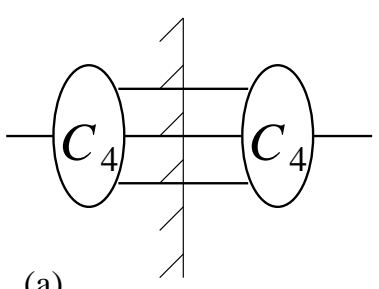

(a)
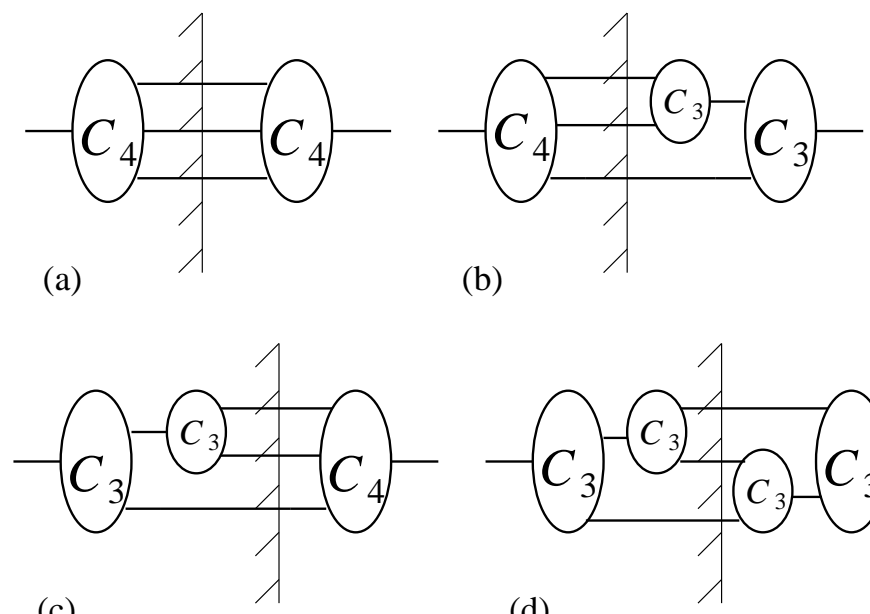

(c)

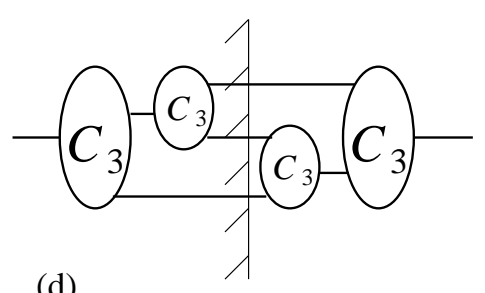

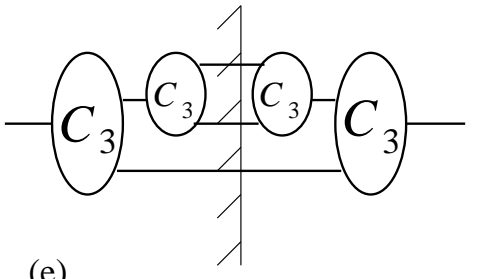

(e)

FIG. 5: Diagrammatic depiction of $\left|M_{4}\right|^{2}$. Here $C_{n}$ denotes the sum of all 1particle irreducible skeleton diagrams with $n$ external legs. Diagrams (a-d) make up $\left.\left\langle k,\left\{l_{k}\right\}\left|\mathcal{M}^{\dagger}\right|\left\{p_{j}\right\}\right\rangle\left\langle\left\{p_{j}\right\}|\mathcal{M}| k,\left\{l_{k}\right\}\right\rangle\right|_{\text {skeleton }}$ while (e) is generated by lower order (in the number of cut momenta) skeleton diagram as shown in Fig. 6.

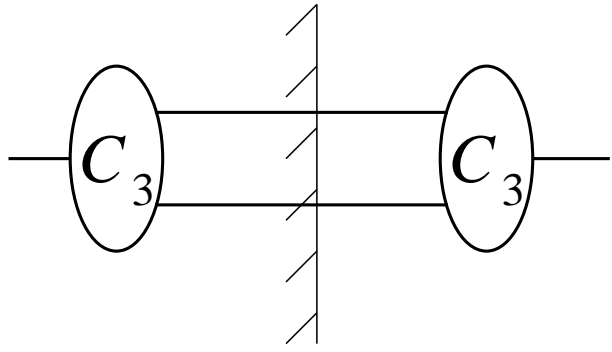

$\Pi_{3}$

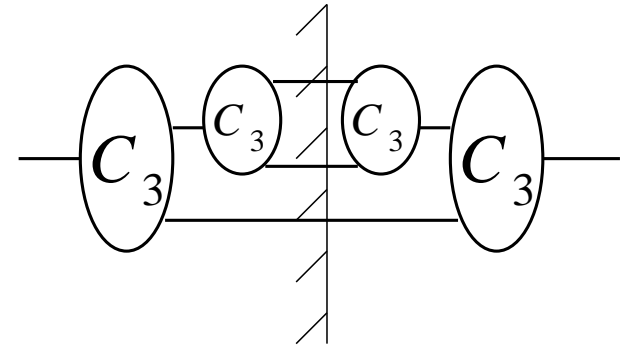

A part of $\Pi_{4}$ that is missing in the skeleton sum

FIG. 6: Lower order (in the number of the cut momenta) skeleton diagram that generate the 2-particle reducible diagram (e) in Fig. [5] when iterated once.

and

$$
d \Gamma^{0}(p)=d \Gamma_{+}^{0}(p)+d \Gamma_{-}^{0}(-p)=\frac{d^{4} p}{(2 \pi)^{4}} G_{12}^{0}(p)
$$

and rewrite Eq.(23) as follows

$$
\Pi_{\text {Boltz }}^{>}(k)=\sum_{n=2}^{\infty} \frac{1}{n !}\left(\int \prod_{i=1}^{n} d \Gamma^{0}\left(l_{i}\right)\right)\left|M_{n+1}\left(k,\left\{l_{i}\right\}_{n}\right)\right|^{2}
$$



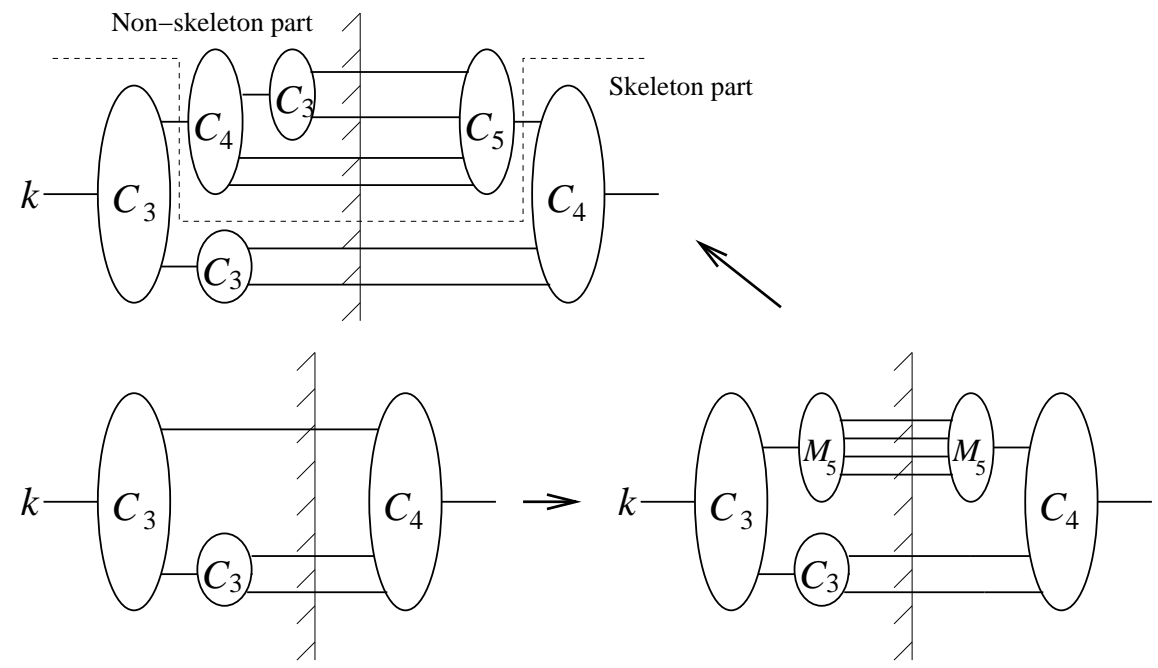

FIG. 7: A nested non-skeleton diagram that contributes to $\left|M_{7}\right|^{2}$. Also shown are the lower order skeleton diagram which generates the nested non-skeleton diagram when iterated with $M_{5}$.

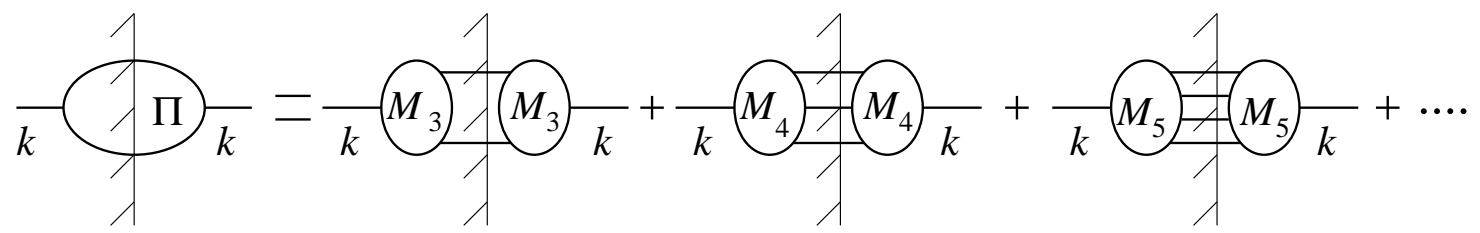

FIG. 8: Graphical representation of Eq.(151).

where $M_{n+1}\left(k,\left\{l_{i}\right\}_{n}\right)$ is the sum of all Feynman diagrams involving $k$ and a set of $n$ external momenta $\left\{l_{i}\right\}_{n}$. Depending on the sign of the energy, an $l_{i}$ can be either an initial-state momentum or a final-state momentum. A graphical representation of Eq.(51) is given in Fig. 8. Note here that a similar result has been obtained in the two-loop case [42]. Using the symmetry of the bosonic multi-particle state, we also rewrite Eq.(46) as

$$
\begin{aligned}
\Pi^{>}(k)= & \sum_{n=0}^{\infty} \sum_{s=0}^{\infty} \frac{1}{n ! s !} \\
& \times \sum_{m=0}^{n} \frac{n !}{m !(n-m) !}\left(\int \prod_{a=1}^{n-m} d \Gamma_{-}^{0}\left(l_{a}\right)\right)\left(\int \prod_{b=1}^{m} \frac{d^{4} \tilde{l}_{b}}{(2 \pi)^{4}} \theta\left(l_{b}^{0}\right) G_{V}\left(\tilde{l}_{b}\right) G_{V}^{*}\left(\tilde{l}_{b}\right) 2 \Pi^{<}\left(\tilde{l}_{b}\right)\right) \\
& \times \sum_{t=0}^{s} \frac{s !}{t !(s-t) !}\left(\int \prod_{c=1}^{s-t} d \Gamma_{+}^{0}\left(p_{c}\right)\right)\left(\int \prod_{d=1}^{t} \frac{d^{4} \tilde{p}_{d}}{(2 \pi)^{4}} \theta\left(p_{d}^{0}\right) G_{V}\left(\tilde{p}_{d}\right) G_{V}^{*}\left(\tilde{p}_{d}\right) 2 \Pi^{>}\left(\tilde{p}_{d}\right)\right) \\
& \times\left.\left\langle k,\left\{l_{a}, \tilde{l}_{b}\right\}_{n}\left|\mathcal{M}^{\dagger}\right|\left\{p_{c}, \tilde{p}_{d}\right\}_{s}\right\rangle\left\langle\left\{p_{c}, \tilde{p}_{d}\right\}_{s}|\mathcal{M}| k,\left\{l_{a}, \tilde{l}_{b}\right\}_{n}\right\rangle\right|_{\text {skeleton }}
\end{aligned}
$$

where the tilde over a momentum variable is a reminder that it is an off-shell momenta. Substituting Eq.(51) into the right hand side of Eq.(52) and shifting some indices, one 
arrives at

$$
\begin{aligned}
& \Pi^{>}(k)=\sum_{n=0}^{\infty} \sum_{m=0}^{\infty} \sum_{t=0}^{\infty} \sum_{s=0}^{\infty} \\
& \times \frac{1}{m ! n !} \int \prod_{a=1}^{n} d \Gamma_{-}^{0}\left(l_{a}\right) \int \prod_{b=1}^{m} \frac{d^{4} \tilde{l}_{b}}{(2 \pi)^{4}} \theta\left(l_{b}^{0}\right) G_{V}\left(\tilde{l}_{b}\right) G_{V}^{*}\left(\tilde{l}_{b}\right) \\
& \times {\left[\sum_{u_{b}=2}^{\infty} \frac{1}{u_{b} !} \int \prod_{i_{u_{b}}=1}^{u_{b}} d \Gamma^{0}\left(l_{i_{u_{b}}}\right)\left|M_{u_{b}+1}\left(\tilde{l}_{b},\left\{l_{i_{u_{b}}}\right\}_{u_{b}}\right)\right|^{2}\right] } \\
& \times \frac{1}{t ! s !} \int \prod_{c=1}^{s} d \Gamma_{+}^{0}\left(p_{c}\right) \int \prod_{d=1}^{t} \frac{d^{4} \tilde{p}_{d}}{(2 \pi)^{4}} \theta\left(p_{d}^{0}\right) G_{V}\left(\tilde{p}_{d}\right) G_{V}^{*}\left(\tilde{p}_{d}\right) \\
& \times\left[\sum_{v_{d}=2}^{\infty} \frac{1}{v_{d} !} \int \prod_{j_{v_{d}}=1}^{v_{d}} d \Gamma^{0}\left(l_{j_{v_{d}}}\right)\left|M_{v_{d}+1}\left(\tilde{p}_{d},\left\{l_{j_{v_{d}}}\right\}_{v_{d}}\right)\right|^{2}\right] \\
& \times\left.\left\langle k,\left\{l_{a}, \tilde{l}_{b}\right\}_{n+m}\left|\mathcal{M}^{\dagger}\right|\left\{p_{c}, \tilde{p}_{d}\right\}_{s+t}\right\rangle\left\langle\left\{p_{c}, \tilde{p}_{d}\right\}_{s+t}|\mathcal{M}| k,\left\{l_{a}, \tilde{l}_{b}\right\}_{n+m}\right\rangle\right|_{\text {skeleton }}
\end{aligned}
$$

What we need to show is that Eq.(51) and Eq.(53) are in fact equivalent.

To show that the two expressions are indeed equivalent, we start with Eq.(51). Consider an $M_{n}$ in Eq.(151). What we would like to do now is to decompose $M_{n}$ in terms of 1-particle irreducible parts. Since Eq.(51) is the result of the skeleton expansion, the lowest order (in the number of legs) $M_{n}$ is $M_{3}$ and has only the 1-particle irreducible part shown in Fig. 9. For $n>3, M_{n}$ can be decomposed into the 1-particle irreducible diagram involving all $n$ particles (denoted by $C_{n}$ ) and diagrams that are combinations of lower order $C_{m}$ 's and $M_{m^{\prime}}$ 's with $m<n$ and $m^{\prime}<n$ as illustrated in Fig. 9. The $M_{m^{\prime}}$ part in turn is made of lower order $C$ 's and $M$ 's, and the iteration continues until no $M$ is left.

Since the iteration starts with $M_{3}=C_{3}$, one can then say that $M_{n}$ consists of all tree-like diagrams but with the vertices replaced by appropriate $C_{m}$ with $m \leq n$. These diagrams, however, are not the tree diagrams of the underlying theory since the tree diagrams in the underlying scalar field theory can only have 3-momentum and 4-momentum vertices while in these tree-like diagrams any $C_{m}$ can play the role of a vertex. As an example, we show graphical representation of this decomposition for $M_{3}, M_{4}$ and $M_{5}$ in Fig. 9.

The diagrams that contribute to $\left|M_{n}\right|^{2}$ can be now generated by summing over all pairs of the tree-like diagrams from $M_{n}$ and $M_{n}^{*}$ and over all possible distinct joinings of the $n-1$ legs (excluding $k$ ) between the chosen pair; see Fig. 5 for an example. Among these joined diagrams, the majority are skeleton diagrams. A non-skeleton diagram results when a tree branch from the $M_{n}$ side fully connects with a tree branch from the $M_{n}^{*}$ side as in the diagram (e) of Fig. 5. These branches could be a primary branch which connects to the root $C$ (where the external momentum $k$ enters) by a single line, or an $r$-th removed 


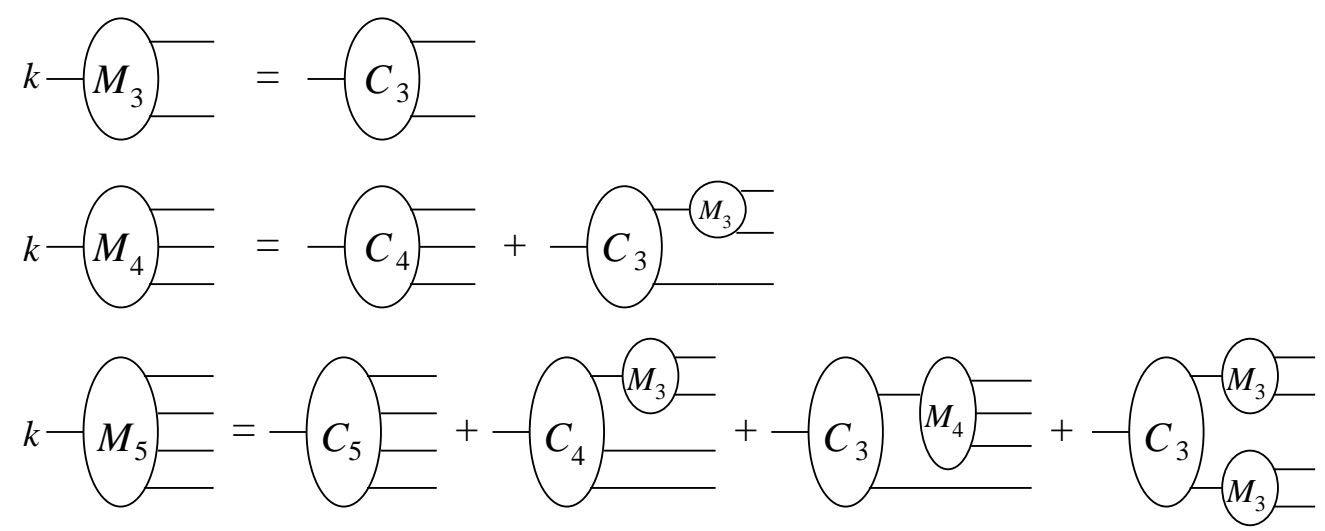

FIG. 9: Decomposition of $M_{n}$ in terms of the 1-particle irreducible part $C_{n}$ and other $C_{m}$ and $M_{m}$ with $m<n$. Here $n$ and $m$ indicate the number of external momenta involved in the process including $k$. For the process involving 3 external momenta, we have $M_{3}=C_{3}$. The $M_{4}$ that appears in the third line can be further expanded using the second line.

branch which needs to go through at least $r$ number of $C$ 's to join with the root $C$. Since all possible tree-like diagrams are being summed over, it is clear that these joined branches sum to $\left|M_{q+1}\right|^{2}$, where $q$ is the number of joined legs. It is also clear that replacing all joined branches with the on-shell part in Eq.(411) reduces the diagram to a lower order skeleton diagram and this reduction is unique. Consequently,

$$
\begin{aligned}
& \left|M_{N+1}\left(k,\left\{l_{i}\right\}_{N}\right)\right|^{2}=\sum_{m=0}^{\infty} \sum_{n=0}^{\infty} \sum_{t=0}^{\infty} \sum_{s=0}^{\infty} \\
& \quad \frac{1}{m ! n !}\left(\int \prod_{b=1}^{m} \frac{d^{4} \tilde{l}_{b}}{(2 \pi)^{4}} \theta\left(l_{b}^{0}\right) G_{V}\left(\tilde{l}_{b}\right) G_{V}^{*}\left(\tilde{l}_{b}\right)\right)\left[\sum_{u_{b}=2}^{\infty} \frac{1}{u_{b} !}\left|M_{u_{b}+1}\left(\tilde{l}_{b},\left\{l_{i_{u_{b}}}\right\}_{u_{b}}\right)\right|^{2}\right] \\
& \quad \times \frac{1}{t ! s !}\left(\int \prod_{d=1}^{t} \frac{d^{4} \tilde{l}_{d}}{(2 \pi)^{4}} \theta\left(\tilde{l}_{d}^{0}\right) G_{V}\left(\tilde{l}_{d}\right) G_{V}^{*}\left(\tilde{l}_{d}\right)\right)\left[\sum_{v_{d}=2}^{\infty} \frac{1}{v_{d} !}\left|M_{v_{d}+1}\left(\tilde{l}_{d},\left\{l_{j_{v_{d}}}\right\}_{v_{d}}\right)\right|^{2}\right] \\
& \quad \times\left.\left\langle k,\left\{l_{a}, \tilde{l}_{b}\right\}_{n+m}\left|\mathcal{M}^{\dagger}\right|\left\{l_{c}, \tilde{l}_{d}\right\}_{s+t}\right\rangle\left\langle\left\{l_{c}, \tilde{l}_{d}\right\}_{s+t}|\mathcal{M}| k,\left\{l_{a}, \tilde{l}_{b}\right\}_{n+m}\right\rangle\right|_{\text {skeleton }} \\
& \quad \times \delta\left(N-n-s-u_{b}-v_{d}\right)
\end{aligned}
$$

with the external momenta given by $\left\{l_{i}\right\}_{N}=\left\{l_{a}, l_{c}, l_{i_{u_{b}}}, l_{j_{v_{d}}}\right\}_{n+s+u_{b}+v_{d}}$ and $\delta(N-n-s-$ $\left.u_{b}-v_{d}\right)$ is a Kronecker- $\delta$. The symmetry factors in Eq. (54) are obtained as follows. One starts with

$$
\left.\frac{1}{(n+m) !(s+t) !}\left\langle k,\left\{l_{i}\right\}_{n+m}\left|\mathcal{M}^{\dagger}\right|\left\{l_{f}\right\}_{s+t}\right\rangle\left\langle\left\{l_{f}\right\}_{s+t}|\mathcal{M}| k,\left\{l_{i}\right\}_{n+m}\right\rangle\right|_{\text {skeleton }}
$$

where the factorial factors accounts for the bosonic symmetry. For the initial momenta $\left\{l_{i}\right\}_{n+m}$, there are $(n+m) ! /(m ! n !)$ number of ways to choose $m$ legs that receives a selfenergy insertion. Combining with the original $1 /(n+m)$ !, this yields the $1 /(m ! n !)$ factor in 
the second line. The $1 /(s ! t !)$ factor in the third line is similarly obtained. The $1 / u_{b}$ ! and $1 / v_{d}$ ! factors come from the fact that the symmetry factor for a diagram is given by the order of the permutation group that leaves the diagram intact [22, 24].

Eq.(54) is exactly the content of Eq.(53). We have thus proved that Eq.(151) is the iterative solution of Eq.(46).

So far, no equilibrium assumption has entered into our derivation except for Eqs.(44) and (45). Hence it is possible to replace the bosonic equilibrium distribution function $n\left(\left|p^{0}\right|\right)$ by its non-equilibrium counterpart $f(X, \mathbf{p})$. Within our quasi-particle approximation, the Boltzmann collision term is given by

$$
\begin{aligned}
& C(X, k)= \\
& \begin{aligned}
\frac{1}{2}[1+f(X, \mathbf{k})] \sum_{s=0}^{\infty} \sum_{n=0}^{\infty} \frac{1}{n ! s !} & \left(\int \prod_{i=1}^{n} \frac{d^{3} l_{i}}{(2 \pi)^{3} 2 E_{i}} f\left(X, \mathbf{l}_{i}\right)\right)\left(\int \prod_{j=1}^{s} \frac{d^{3} p_{j}}{(2 \pi)^{3} 2 E_{j}}\left[1+f\left(X, \mathbf{p}_{j}\right)\right]\right) \\
& \times\left|\left\langle k,\left\{p_{j}\right\}_{s}|\mathcal{M}|\left\{l_{i}\right\}_{n}\right\rangle\right|^{2} \\
-\frac{1}{2} f(X, \mathbf{k}) \sum_{s=0}^{\infty} \sum_{n=0}^{\infty} \frac{1}{n ! s !}\left(\int\right. & \left.\prod_{i=1}^{n} \frac{d^{3} l_{i}}{(2 \pi)^{3} 2 E_{i}} f\left(X, \mathbf{l}_{i}\right)\right)\left(\int \prod_{j=1}^{s} \frac{d^{3} p_{j}}{(2 \pi)^{3} 2 E_{j}}\left[1+f\left(X, \mathbf{p}_{j}\right)\right]\right) \\
\times & \left|\left\langle\left\{p_{j}\right\}_{s}|\mathcal{M}| k,\left\{l_{i}\right\}_{n}\right\rangle\right|^{2}
\end{aligned}
\end{aligned}
$$

where $\left|\left\langle\left\{p_{j}\right\}|\mathcal{M}| k,\left\{l_{i}\right\}\right\rangle\right|^{2}$ is the full scattering matrix squared in vacuum with zero temperature masses replaced by thermal masses. The sum $n+s$ should, of course, be greater than or equal to 2 and the energy-momentum conserving $\delta$-function is absorbed in the definition of matrix element squared. This result is similar to the one in Ref. [24], with the difference that in Ref. 24] only tree diagrams are included in the calculation of $|M|^{2}$. The analysis in Ref. 24] also did not fully include resummed propagators.

The main feature of Eq.(56) is that it is naturally expressed in terms of full scattering matrix elements. They can be evaluated theoretically using perturbation theory or by using data from experiments. Note also that all the information about the theory is in the matrix elements. Therefore, the expressions of the gain and loss rates should be valid for any interacting theory of a real scalar field. In the case of $\phi^{3}$ and $\phi^{4}$, it reproduces the results of 16] for 1,2,3,4-loop contributions at tree level. In principle, our analysis could be generalized to gauge theories but the preservation of gauge invariance must be carefully dealt with.

\section{DISCUSSIONS AND CONCLUSIONS}

Equations Eq.(23) and (56) are our main results in this paper. Namely, we have shown that the Wightman self-energy can be approximately expressed in terms of the thermally averaged full scattering matrix elements. We then used this fact to express the Boltzmann 
collision term to all orders. Equations Eq.(23) and (156) are often used in various calculations such as in-medium particle property calculations [43, 44, 45, 46, 47, 48, 49] and MonteCarlo simulations of many-body systems; but, as far as we know, a systematic derivation has been lacking. Within our quasi-particle approximation, our result is valid for all orders of perturbation theory and any number of participating particles.

Our final results are quite independent of the details of the underlying theory because they involve only the scattering matrix elements. Even though our discussions and results are for real scalar theories, they can in principle be generalized to more complicated theories such as gauge theories. This is clearly the subject of a future work.

An advantage of having such an all-orders formulation is that these matrix elements do not have to be calculated from first principles; instead, they can be measured in vacuum and used as an input for the in-medium calculation. Also, having derived Eq.(156), we can use it as a basis for Monte-Carlo simulations of many-body systems with confidence.

While obtaining our main results, we have made some approximations. Here we would like to list them and comment on them.

(i) Approximations in deriving the Kadanoff-Baym equation.

These approximations are valid in the weakly inhomogeneous system and the weak interaction strength limit. Since the Kadanoff-Baym equation has been much discussed in the literature (see for example [52, 54, 55, 56] for some discussion in the context of 2PI effective action methods), we have little more to add to this point.

(ii) Quasi-particle approximation of the spectral density, Eq.(41).

This is somewhat different than the quasi-particle ansatz made in [16, 24] in that we have included the continuum part of the spectral density. This approximation should be valid under the same conditions that the Kadanoff-Baym equation is valid. To go beyond that, we need to repeat our analysis using the off-shell transport theory developed in Refs. [32, 33, 50, 51, 52, 53]. This is clearly outside the scope of the present paper.

(iii) Ignoring thermal corrections to the interaction vertices, Eq.(43).

Our quasi-particle ansatz could easily accomodate the in-medium mass, but we had to make an approximation where we ignored thermal vertex corrections. Physically, this amounts to ignoring interference between the purely in-medium processes and processes in which the external momentum $k$ is involved. In many physical situations, ignoring these corrections can lead to serious inconsistencies. In the case of real scalar theories, this is not much of a concern. But when generalizing to gauge theories, 
thermal vertex corrections must be taken into account. For instance, in hard thermal loop calculations in hot QCD, it is essential to include thermal corrections to both vertices and self-energies to preserve gauge symmetry.

It should be possible generalize our formulation to include thermal vertex corrections, but we will have to pay a price: the scattering matrix elements need to include disconnected pieces so that both diagrams in Fig. 1 1 are included. Suppose we denote the sum of all cut in-medium diagrams (the analogue of the vacuum bubbles) by $W_{T}$. In vaccum, this is zero because the vacuum cannot spontaneously generate propagating particles. In a medium, however, $W_{T}$ encodes contributions from the independent scattering processes occuring in the medium. Just like the vacuum phase, the missing diagrams in $\mathcal{K}_{A}$ can be added by multiplying the self-energy by $e^{W_{T}}$. But this extra factor is not a part of the self-energy. Hence, we must compensate it with an additional factor of $e^{-W_{T}}$. Whether we can repeat our analysis in this way is currently under investigation.

In summary, we have greatly extended our previous work 23] and a similar work by Weinstock 24] to derive the Boltzmann collision term from the Kadanoff-Baym equation as fully as possible. Along the way, we have identified the approximations that have to be made and possible remedies to include the ignored effects. Work along this line is continuing. It is hoped that this work lays a firm foundation for the inclusion of many-body scattering processes in Monte-Carlo simulations. We also mention that it is possible to generalize this result to include strong classical sources. First steps in this direction have been taken in Ref. [57] and work along these lines is currently in progress.

\section{Acknowledgments}

We thank S. Turbides for useful discussions and A. Berndsen for a careful proofreading of the manuscript. This work was supported in part by the Natural Sciences and Engineering Research Council of Canada, and in part, for S.J., by le Fonds Nature et Technologies du Québec. S.J. also thanks RIKEN BNL Center and U.S. Department of Energy [DE-AC0298CH10886] for providing facilities essential for the completion of this work.

[1] L. D. McLerran and R. Venugopalan, Phys. Rev. D 49, 2233 (1994) arXiv:hep-ph/9309289.

[2] L. D. McLerran and R. Venugopalan, Phys. Rev. D 49, 3352 (1994) arXiv:hep-ph/9311205.

[3] L. McLerran, Nucl. Phys. A 752, 355 (2005). 
[4] R. Venugopalan, Eur. Phys. J. C 43, 337 (2005) arXiv:hep-ph/0502190.

[5] D. Kharzeev and M. Nardi, Phys. Lett. B 507, 121 (2001) arXiv:nucl-th/0012025.

[6] D. Kharzeev, E. Levin and M. Nardi, Phys. Rev. C 71, 054903 (2005) arXiv:hep-ph/0111315.

[7] D. Kharzeev, E. Levin and M. Nardi, Nucl. Phys. A 730, 448 (2004) [Erratum-ibid. A 743, 329 (2004)] arXiv:hep-ph/0212316.

[8] J. Schwinger, J. Math. Phys. 2, 407 (1961).

[9] L. V. Keldysh, Sov. Phys. JETP 20, 1018 (1964).

[10] S. Jeon, Phys. Rev. D 52, 3591 (1995) [arXiv: hep-ph/9409250.

[11] S. Jeon and L. G. Yaffe, Phys. Rev. D 53, 5799 (1996) [arXiv: hep-ph/9512263.

[12] P. Arnold, G. D. Moore and L. G. Yaffe, JHEP 0011, 001 (2000) [arXiv: hep-ph/0010177.

[13] P. Arnold, G. D. Moore and L. G. Yaffe, JHEP 0305, 051 (2003) [arXiv: hep-ph/0302165.

[14] P. Arnold, G. D. Moore and L. G. Yaffe, JHEP 0301, 030 (2003) [arXiv: hep-ph/0209353.

[15] L. P. Kadanoff and P. C. Martin, Ann. Phys. NY 24, 419 (1963).

[16] M. E. Carrington and S. Mrówczyński, Phys. Rev. D 71065007 (2005) [arXiv: hep-ph/0406097.

[17] S. Mrowczynski and P. Danielewicz, Nucl. Phys. B 342, 345 (1990).

[18] R. Baier et al., Phys. Lett. B 502, 51 (2001) [arXiv: hep-ph/0009237.

[19] D. K. Srivastava and K. Geiger, Nucl. Phys. A 647, 136 (1999) [arXiv: nucl-th/9806050.

[20] S. M. H. Wong, arXiv: hep-ph/0404222.

[21] Z. Xu and C. Greiner, Phys. Rev. C 71, 064901 (2005) arXiv:hep-ph/0406278.

[22] S. Jeon and P. J. Ellis, Phys. Rev. D 58, 045013 (1998) [arXiv: hep-ph/9802246.

[23] J.-S. Gagnon, F. Fillion-Gourdeault and S. Jeon, Quark Matter 2005 proceedings, arXiv: hep-ph/0510367.

[24] S. Weinstock, Phys. Rev. D 73, 025005 (2006) arXiv:hep-ph/0510417.

[25] H. T. Elze and U. W. Heinz, Phys. Rept. 183, 81 (1989).

[26] S. Mrowczynski, Phys. Rev. D 56, 2265 (1997). arXiv:hep-th/9702022.

[27] L.P. Kadanoff and G. Baym, Quantum Statistical Mechanics (Benjamin, New York (1962)).

[28] S.R. de Groot, W.A. van Leeuwen and Ch.G. van Weert, Relativistic Kinetic Theory (NorthHolland, Amsterdam (1980)).

[29] R. L. Kobes and G. W. Semenoff, Nucl. Phys. B 260, 714 (1985); Nucl. Phys. B 272, 329 (1986).

[30] G. 't Hooft and M. Veltman, "Diagrammar", CERN Yellow Report 73-9 (1973).

[31] S. Weinberg, The Quantum Theory of Fields (Cambridge University Press, Cambridge, (2002)).

[32] N. P. Landsman and Ch. G. van Weert, Phys. Rep. 145, 141 (1987). 
[33] N. P. Landsman, Annals Phys. 186, 141 (1988).

[34] T. Altherr and D. Seibert, Phys. Lett. B 333, 149 (1994) [arXiv: hep-ph/9405396.

[35] T. Altherr, Phys. Lett. B 341, 325 (1995) [arXiv: hep-ph/9407249.

[36] P. F. Bedaque, Phys. Lett. B 344, 23 (1995) [arXiv: hep-ph/9410415.

[37] C. Greiner and S. Leupold, Annals Phys. 270, 328 (1998) [arXiv: hep-ph/9802312.

[38] C. Greiner and S. Leupold, Eur. Phys. J. C 8, 517 (1999) [arXiv: hep-ph/9804239.

[39] E. Braaten and R. D. Pisarski, Nucl. Phys. B 337, 569 (1990).

[40] J. Frenkel and J. C. Taylor, Nucl. Phys. B 334, 199 (1990).

[41] G. Aarts and J. Berges, Phys. Rev. D 64, 105010 (2001) arXiv:hep-ph/0103049.

[42] A. Majumder and C. Gale, Phys. Rev. C 65, 055203 (2002) arXiv:hep-ph/0111181.

[43] C. Gale, Eur. Phys. J. C 43, 381 (2005) arXiv:hep-ph/0504103.

[44] S. Mallik, Eur. Phys. J. C 24, 143 (2002) arXiv:hep-th/0108139.

[45] V. L. Eletsky, M. Belkacem, P. J. Ellis and J. I. Kapusta, Phys. Rev. C 64, 035202 (2001) arXiv:nucl-th/0104029.

[46] J. I. Kapusta and S. M. H. Wong, Phys. Rev. D 64, 045008 (2001) arXiv:hep-th/0103065.

[47] G. Amelino-Camelia and J. I. Kapusta, Phys. Lett. B 465, 291 (1999) arXiv:hep-ph/9907508.

[48] S. Gao, C. Gale, C. Ernst, H. Stoecker and W. Greiner, arXiv:nucl-th/9812059.

[49] V. L. Eletsky and J. I. Kapusta, arXiv:nucl-th/9810052.

[50] S. Leupold, Nucl. Phys. A 672, 475 (2000) arXiv:nucl-th/9909080.

[51] Y. B. Ivanov, J. Knoll and D. N. Voskresensky, Nucl. Phys. A 672, 313 (2000) arXiv:nucl-th/9905028.

[52] S. Juchem, W. Cassing and C. Greiner, Nucl. Phys. A 743, 92 (2004) arXiv:nucl-th/0401046.

[53] W. Cassing and S. Juchem, Nucl. Phys. A 677, 445 (2000) arXiv:nucl-th/0003002.

[54] M. Lindner and M. M. Muller, arXiv:hep-ph/0512147.

[55] J. Knoll, Y. B. Ivanov and D. N. Voskresensky, Annals Phys. 293, 126 (2001) arXiv:nucl-th/0102044.

[56] Y. B. Ivanov, J. Knoll and D. N. Voskresensky, Nucl. Phys. A 657, 413 (1999) arXiv:hep-ph/9807351.

[57] F. Gelis and R. Venugopalan, arXiv: hep-ph/0601209.

\section{APPENDIX A: $2 \leftrightarrow 2$ PROCESS EXAMPLE}

In this example, we illustrate how the iteration procedure described in Sect. IV adds necessary diagrams to get full scattering matrix elements using the $g \phi^{3}$ scalar theory. We will look at the first non-trivial and kinematically allowed process: the $2 \rightarrow 2$ process. We start from the skeleton expansion of the Wightman self-energy. To lowest order, the expan- 

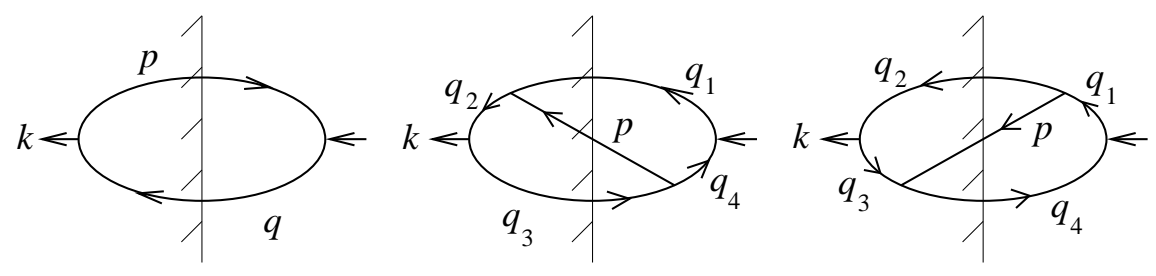

FIG. 10: Lowest order skeleton diagrams in the $g \phi^{3}$ theory.

sion includes the diagrams in Fig. 10, The mathematical expression corresponding to the Wightman self-energy is

$$
\begin{aligned}
\Pi^{>}(k)= & \frac{g^{2}}{2} \int \frac{d^{4} p}{(2 \pi)^{4}} \frac{d^{4} q}{(2 \pi)^{4}} G_{21}(p) G_{12}(q)(2 \pi)^{4} \delta^{4}(k+p-q) \\
& +\frac{g^{4}}{2} \int \prod_{i=1}^{4} \frac{d^{4} q_{i}}{(2 \pi)^{4}} \frac{d^{4} p}{(2 \pi)^{4}}(2 \pi)^{12} \delta^{4}\left(k+q_{4}-q_{1}\right) \delta^{4}\left(q_{1}-p-q_{2}\right) \delta^{4}\left(p+q_{3}-q_{4}\right) \\
& \times\left[G_{12}\left(q_{1}\right) G_{22}\left(q_{2}\right) G_{21}\left(q_{3}\right) G_{11}\left(q_{4}\right) G_{21}(p)+G_{11}\left(q_{1}\right) G_{12}\left(q_{2}\right) G_{22}\left(q_{3}\right) G_{21}\left(q_{4}\right) G_{12}(p)\right] \\
& + \text { other cuts of } \mathcal{O}\left(g^{4}\right)+\mathcal{O}\left(g^{6}\right)
\end{aligned}
$$

where 'other cuts' do not contribute to the 2 to 2 process.

Neglecting the thermal phase space factor inside $G_{11}(k)$ and $G_{22}(k)$ yields

$$
\begin{aligned}
\Pi^{>}(k)= & \frac{g^{2}}{2} \int \frac{d^{4} p}{(2 \pi)^{4}} \frac{d^{4} q}{(2 \pi)^{4}} G_{21}(p) G_{12}(q)(2 \pi)^{4} \delta^{4}(k+p-q) \\
& +\frac{g^{4}}{2} \int \prod_{i=1}^{4} \frac{d^{4} q_{i}}{(2 \pi)^{4}} \frac{d^{4} p}{(2 \pi)^{4}}(2 \pi)^{12} \delta^{4}\left(k+q_{4}-q_{1}\right) \delta^{4}\left(q_{1}-p-q_{2}\right) \delta^{4}\left(p+q_{3}-q_{4}\right) \\
& \times\left[G_{12}\left(q_{1}\right) G_{V}^{*}\left(q_{2}\right) G_{21}\left(q_{3}\right) G_{V}\left(q_{4}\right) G_{21}(p)+G_{V}\left(q_{1}\right) G_{12}\left(q_{2}\right) G_{V}^{*}\left(q_{3}\right) G_{21}\left(q_{4}\right) G_{12}(p)\right]
\end{aligned}
$$

where we defined

$$
G_{V}(k)=\frac{i}{k^{2}-m_{T}+i\left|\Pi_{I}^{V}\right|}
$$

Let's consider the first term.

$$
\Pi_{(1)}^{>}=\frac{g^{2}}{2} \int \frac{d^{4} p}{(2 \pi)^{4}} \frac{d^{4} q}{(2 \pi)^{4}} G_{21}(p) G_{12}(q)(2 \pi)^{4} \delta^{4}(k+p-q)
$$

Using the fact that the spectral density is odd $(\rho(-p)=-\rho(p))$ and by making a suitable 
change of variable, this becomes

$$
\begin{aligned}
& \Pi_{(1)}^{>}(k)=\frac{g^{2}}{2} \int \frac{d^{4} p}{(2 \pi)^{4}} \frac{d^{4} q}{(2 \pi)^{4}} \theta\left(p^{0}\right) \rho(p) \theta\left(q^{0}\right) \rho(q)(2 \pi)^{4} \\
& \times\{ {\left[1+n\left(\left|p^{0}\right|\right)\right]\left[1+n\left(\left|q^{0}\right|\right)\right] \delta^{4}(k-p-q) } \\
&+\left[1+n\left(\left|p^{0}\right|\right)\right] n\left(\left|q^{0}\right|\right) \delta^{4}(k-p+q) \\
&+n\left(\left|p^{0}\right|\right)\left[1+n\left(\left|q^{0}\right|\right)\right] \delta^{4}(k+p-q) \\
&\left.+n\left(\left|p^{0}\right|\right) n\left(\left|q^{0}\right|\right) \delta^{4}(k+p+q)\right\}
\end{aligned}
$$

Each terms corresponds to a lowest order transition amplitude between off-shell states coming from the skeleton expansion as defined previously in $\mathcal{K}_{A}$.

The second term is

$$
\begin{aligned}
\Pi_{(2)}^{>}(k)= & \frac{g^{4}}{2} \int \prod_{i=1}^{4} \frac{d^{4} q_{i}}{(2 \pi)^{4}} \frac{d^{4} p}{(2 \pi)^{4}}(2 \pi)^{12} \delta^{4}\left(k+q_{4}-q_{1}\right) \delta^{4}\left(q_{1}-p-q_{2}\right) \delta^{4}\left(p+q_{3}-q_{4}\right) \\
& \times\left[G_{12}\left(q_{1}\right) G_{V}^{*}\left(q_{2}\right) G_{21}\left(q_{3}\right) G_{V}\left(q_{4}\right) G_{21}(p)+G_{V}\left(q_{1}\right) G_{12}\left(q_{2}\right) G_{V}^{*}\left(q_{3}\right) G_{21}\left(q_{4}\right) G_{12}(p)\right]
\end{aligned}
$$

We can use the properties of the spectral function and the explicit expression of the cut propagators to write the preceding equation as

$$
\begin{aligned}
\Pi_{(2)}^{>}(k)= & \frac{g^{4}}{2} \int \frac{d^{4} q_{1}}{(2 \pi)^{4}} \frac{d^{4} q_{2}}{(2 \pi)^{4}} \frac{d^{4} q_{3}}{(2 \pi)^{4}}(2 \pi)^{4} \delta^{4}\left(k+q_{1}-q_{2}-q_{3}\right) \\
& \times \theta\left(q_{1}^{0}\right) \theta\left(q_{2}^{0}\right) \theta\left(q_{3}^{0}\right) \rho\left(q_{1}\right) \rho\left(q_{2}\right) \rho\left(q_{3}\right) n\left(\left|q_{1}^{0}\right|\right)\left[1+n\left(\left|q_{2}^{0}\right|\right)\right]\left[1+n\left(\left|q_{3}^{0}\right|\right)\right] \\
& \times\left[G_{V}^{*}\left(q_{3}-q_{1}\right) G_{V}\left(q_{2}-q_{1}\right)+G_{V}^{*}\left(q_{3}+q_{2}\right) G_{V}\left(q_{2}-q_{1}\right)+G_{V}^{*}\left(q_{3}-q_{1}\right) G_{V}\left(q_{2}+q_{3}\right)\right. \\
& \left.+G_{V}^{*}\left(q_{2}-q_{1}\right) G_{V}\left(q_{3}-q_{1}\right)+G_{V}^{*}\left(q_{2}-q_{1}\right) G_{V}\left(q_{2}+q_{3}\right)+G_{V}^{*}\left(q_{3}+q_{2}\right) G_{V}\left(q_{3}-q_{1}\right)\right] \\
& +(\text { other processes) }
\end{aligned}
$$

Each term corresponds to a different channel of a $2 \leftrightarrow 2$ scattering process. The neglected terms lead to other processes like $1 \leftrightarrow 3$. If we now make the lowest order quasi-particle ansatz, $\rho(p) \rightarrow(2 \pi) \delta\left(p^{2}-m_{T}^{2}\right)$, the above expression can be written as

$$
\begin{aligned}
\Pi_{(2)}^{>}(k)= & \frac{1}{2} \int \frac{d^{3} q_{1}}{(2 \pi)^{3} 3 E_{1}} \frac{d^{3} q_{2}}{(2 \pi)^{3} 3 E_{3}} \frac{d^{3} q_{3}}{(2 \pi)^{3} 3 E_{3}}(2 \pi)^{4} \delta^{4}\left(k+q_{1}-q_{2}-q_{3}\right) \\
& \times n\left(E_{1}\right)\left[1+n\left(E_{2}\right)\right]\left[1+n\left(E_{3}\right)\right] \\
& \times\left[M_{t}^{*} M_{u}+M_{s}^{*} M_{u}+M_{t}^{*} M_{s}+M_{u}^{*} M_{t}+M_{u}^{*} M_{s}+M_{s}^{*} M_{t}\right] \\
& +(\text { other processes })
\end{aligned}
$$

Here $M_{s}, M_{t}, M_{u}$ represent the scattering amplitude in the $s, t . u$ channel, respectively. The terms in the square bracket is not yet the full scattering element squared since the square 
terms are missing. We only have the interference terms. The squared terms should come from iterating Eq.(A5). We can expand the spectral density for this one-loop diagram using Eq.(41) and keep only the relevant terms for $2 \rightarrow 2$ process at lowest order. This yields, in addition to the pure one-loop contribution,

$$
\begin{aligned}
\Pi_{(1-2)}^{>}(k)= & \frac{g^{2}}{2} \int \frac{d^{4} p}{(2 \pi)^{4}} \frac{d^{4} q}{(2 \pi)^{4}} \theta\left(p^{0}\right) \theta\left(q^{0}\right)(2 \pi)^{4} \\
& \times\left[2(2 \pi) \delta\left(p^{2}-m_{T}^{2}\right) G_{V}^{*}(q) \Pi_{I}(q) G_{V}(q)+2(2 \pi) \delta\left(q^{2}-m_{T}^{2}\right) G_{V}^{*}(p) \Pi_{I}(p) G_{V}(p)\right] \\
& \times\left\{\left[1+n\left(\left|p^{0}\right|\right)\right]\left[1+n\left(\left|q^{0}\right|\right)\right] \delta^{4}(k-p-q)+2\left[1+n\left(\left|p^{0}\right|\right)\right] n\left(\left|q^{0}\right|\right) \delta^{4}(k-p+q)\right. \\
& \left.+n\left(\left|p^{0}\right|\right) n\left(\left|q^{0}\right|\right) \delta^{4}(k+p+q)\right\} \\
& + \text { (other processes) }
\end{aligned}
$$

Upon using Eqs.(44) and (45) this becomes

$$
\begin{aligned}
\Pi_{(1)}^{>}(k)= & \frac{g^{2}}{2} \int \frac{d^{4} p}{(2 \pi)^{4}} \frac{d^{4} q}{(2 \pi)^{4}} \theta\left(p^{0}\right) \theta\left(q^{0}\right)(2 \pi)^{4} \\
\times & \left\{\left[1+n\left(\left|p^{0}\right|\right)\right](2 \pi) \delta\left(p^{2}-m_{T}^{2}\right) G_{V}^{*}(q) \Pi^{>}(q) G_{V}(q) \delta^{4}(k-p-q)\right. \\
& +\left[1+n\left(\left|p^{0}\right|\right)\right](2 \pi) \delta\left(p^{2}-m_{T}^{2}\right) G_{V}^{*}(q) \Pi^{<}(q) G_{V}(q) \delta^{4}(k-p+q) \\
& +n\left(\left|p^{0}\right|\right)(2 \pi) \delta\left(p^{2}-m_{T}^{2}\right) G_{V}^{*}(q) \Pi^{>}(q) G_{V}(q) \delta^{4}(k-q+p) \\
& \left.\left.+n\left(\left|p^{0}\right|\right)(2 \pi) \delta\left(p^{2}-m_{T}^{2}\right) G_{V}^{*} q\right) \Pi^{<}(q) G_{V}(q) \delta^{4}(k+p+q)\right\} \\
& + \text { (other processes })
\end{aligned}
$$

The last equation is a self-consistent equation for the self-energy. To solve it, we can use an iteration procedure. Since we are considering only the $2 \rightarrow 2$ processes to the lowest order, this means in the right hand side we should replace $\Pi^{>},<(q)$ with the purely one-loop result

$$
\begin{aligned}
\Pi_{(1-1)}^{>}(k)= & \frac{g^{2}}{2} \int \frac{d^{4} p}{(2 \pi)^{4}} \frac{d^{4} q}{(2 \pi)^{4}} \theta\left(p^{0}\right) \theta\left(q^{0}\right)(2 \pi)^{6} \delta\left(p^{2}-m_{T}^{2}\right) \delta\left(q^{2}-m_{T}^{2}\right) \\
& \times\left\{\left[1+n\left(\left|p^{0}\right|\right)\right]\left[1+n\left(\left|q^{0}\right|\right)\right] \delta^{4}(k-p-q)\right. \\
& +2\left[1+n\left(\left|p^{0}\right|\right)\right] n\left(\left|q^{0}\right|\right) \delta^{4}(k-p+q) \\
& \left.+n\left(\left|p^{0}\right|\right) n\left(\left|q^{0}\right|\right) \delta^{4}(k+p+q)\right\}
\end{aligned}
$$

There is an analogous equation for $\Pi_{(1-1)}^{<}(k)$. Upon substitution, Eq. (A10) can be shown to contain the missing square terms

$$
\begin{aligned}
\Pi_{(1)}^{>}(k)= & \frac{1}{2} \int \frac{d^{3} q_{1}}{(2 \pi)^{3} 2 E_{1}} \frac{d^{3} q_{2}}{(2 \pi)^{3} 2 E_{2}} \frac{d^{3} q_{3}}{(2 \pi)^{3} 2 E_{3}}(2 \pi)^{4} \delta^{4}\left(k+q_{1}-q_{2}-q_{3}\right) \\
& \times n\left(E_{1}\right)\left[1+n\left(E_{2}\right)\right]\left[1+n\left(E_{3}\right)\right]\left[M_{u}^{*} M_{u}+M_{s}^{*} M_{s}+M_{t}^{*} M_{t}\right] \\
& + \text { (other processes) }
\end{aligned}
$$


If we combine our two answers, we get

$$
\begin{aligned}
\Pi^{>}(k)= & \Pi_{(1)}^{>}(k)+\Pi_{(2)}^{>}(k) \\
= & \frac{1}{2} \int \frac{d^{3} q_{1}}{(2 \pi)^{3} 2 E_{1}} \frac{d^{3} q_{2}}{(2 \pi)^{3} 2 E_{2}} \frac{d^{3} q_{3}}{(2 \pi)^{3} 2 E_{3}}(2 \pi)^{4} \delta^{4}\left(k+q_{1}-q_{2}-q_{3}\right) \\
& \times n\left(E_{1}\right)\left[1+n\left(E_{2}\right)\right]\left[1+n\left(E_{3}\right)\right]\left|M_{u}+M_{s}+M_{t}\right|^{2}
\end{aligned}
$$

This is in accordance with our general result expressed in equation (23). 